\title{
The Korteweg-de Vries equation on an interval
}

Cite as: J. Math. Phys. 60, 051507 (2019); https://doi.org/10.1063/1.5080366

Submitted: 07 November 2018 . Accepted: 14 April 2019 . Published Online: 08 May 2019

(D. Alexandrou Himonas, Dionyssios Mantzavinos, and (Dangchi Yan

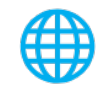

View Online

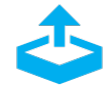

Export Citation

\section{ARTICLES YOU MAY BE INTERESTED IN}

The Cauchy problem for the 1-D Gurevich-Zybin system

Journal of Mathematical Physics 60, 051509 (2019); https://doi.org/10.1063/1.5068755

Vector solutions for two coupled Schrödinger equations on Riemannian manifolds

Journal of Mathematical Physics 60, 051502 (2019); https://doi.org/10.1063/1.5100021

Global existence of the self-interacting scalar field in the de Sitter universe

Journal of Mathematical Physics 60, 051503 (2019); https://doi.org/10.1063/1.5082653

\section{Journal of}

Mathematical Physics

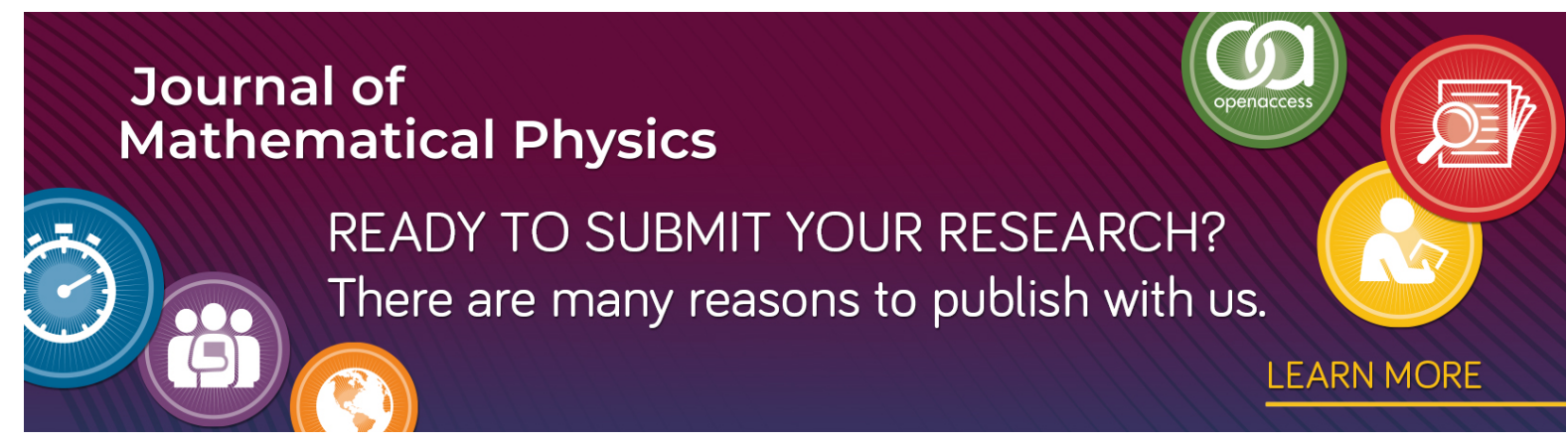

READY TO SUBMIT YOUR RESEARCH?

There are many reasons to publish with us. 


\title{
The Korteweg-de Vries equation on an interval
}

\author{
Cite as: J. Math. Phys. 60, 051507 (2019); doi: 10.1063/1.5080366 \\ Submitted: 7 November 2018 • Accepted: 14 April 2019 • \\ Published Online: 8 May 2019
}

\begin{abstract}
A. Alexandrou Himonas, ${ }^{1, a)}$ (D) Dionyssios Mantzavinos, ${ }^{2, b)}$ and Fangchi Yan ${ }^{1, c)}$ (D)
AFFILIATIONS

${ }^{1}$ Department of Mathematics, University of Notre Dame, Notre Dame, Indiana 46556, USA

${ }^{2}$ Department of Mathematics, University of Kansas, Lawrence, Kansas 66045, USA
\end{abstract}

\author{
a) Author to whom correspondence should be addressed: himonas.1@nd.edu. \\ b)E-mail: mantzavinos@ku.edu \\ ${ }^{c)}$ E-mail: fyan1@nd.edu
}

\begin{abstract}
The initial-boundary value problem (IBVP) for the Korteweg-de Vries (KdV) equation on an interval is studied by extending a novel approach recently developed for the well-posedness of the KdV on the half-line, which is based on the solution formula produced via Fokas' unified transform method for the associated forced linear IBVP. Replacing in this formula the forcing by the nonlinearity and using data in Sobolev spaces suggested by the space-time regularity of the Cauchy problem of the linear KdV gives an iteration map for the IBVP which is shown to be a contraction in an appropriately chosen solution space. The proof relies on key linear estimates and a bilinear estimate similar to the one used for the KdV Cauchy problem by Kenig, Ponce, and Vega.
\end{abstract}

Published under license by AIP Publishing. https://doi.org/10.1063/1.5080366

\section{INTRODUCTION AND RESULTS}

We consider the following initial-boundary value problem (IBVP) for the Korteweg-de Vries (KdV) equation on an interval

$$
\begin{aligned}
& \partial_{t} u+\partial_{x}^{3} u+u \partial_{x} u=0, \quad 0<x<\ell, 0<t<T, \\
& u(x, 0)=u_{0}(x), \\
& u(0, t)=g_{0}(t), \quad u(\ell, t)=h_{0}(t), \quad u_{x}(\ell, t)=h_{1}(t),
\end{aligned}
$$

where $T<1$, and prove its well-posedness for initial data $u_{0}(x)$ in the Sobolev space $H_{x}^{s}(0, \ell), \frac{3}{4}<s<1$, and boundary data suggested by the time regularity of the Cauchy problem of the linear KdV, namely, $g_{0}(t), h_{0}(t)$ in the Sobolev space $H_{t}^{\frac{s+1}{3}}(0, T)$ and $h_{1}(t)$ in the Sobolev space $H_{t}^{\frac{s}{3}}(0, T)$. For this, we develop a natural extension of a novel approach recently introduced for the well-posedness of KdV on the halfline ${ }^{20}$ which is based on the solution formula produced via Fokas' unified transform method (UTM) for the associated linear IBVP. Using the solution formula obtained by the Fokas method on an interval, we define an iteration map and show that it is a contraction on an appropriately chosen solution space by deriving the needed linear estimates and a bilinear estimate analogous to the one used for the KdV on the line by Kenig, Ponce, and Vega.

The KdV equation has a long and celebrated history in both mathematics and physics. It was first derived by Boussinesq in $1877^{10}$ as a model for long waves propagating on shallow water. Boussinesq's main motivation was to provide a theoretical explanation of Scott Russell's observation of the "great wave of translation," nowadays simply known as soliton. ${ }^{45}$ Indeed, the KdV equation (1.1a) admits several traveling wave solutions including the two-parameter family of solitons

$$
u(x, t)=3 c \operatorname{sech}^{2}\left[\frac{\sqrt{c}}{2}(x-c t-b)\right]
$$

$c$ being the speed of propagation and $b$ being an arbitrary constant. In fact, Korteweg and de Vries, who rederived KdV in $1895,^{38}$ showed that the solitons (1.2) arise from a two-parameter family of periodic traveling wave solutions described by elliptic functions known as 
cnoidal waves in the limit of their Jacobi elliptic modulus tending to one. The study of KdV remained dormant for several decades, until the 1965 numerical experiments of Zabusky and Kruskal ${ }^{48}$ for the case of periodic boundary conditions, which indicated that soliton solutions of $\mathrm{KdV}$ interact almost linearly, preserving their shape and speed and experiencing merely a phase shift due to the collision. (Zabusky and Kruskal were actually the ones who came up with the name "soliton.")

The initial value problem (IVP) for KdV has been studied extensively and via different approaches. After the renewed attention sparked by the work of Ref. 48, Gardner et al. ${ }^{27}$ solved the IVP for KdV on the line for data of sufficient smoothness and decay by introducing the celebrated inverse scattering transform, which relies on the integrability of KdV and, more precisely, on the Lax pair formulation

$$
\begin{aligned}
& \mu_{x x}+\left(\frac{1}{6} u+k^{2}\right) \mu=0, \\
& \mu_{t}+\left(\frac{1}{3} u-4 k^{2}\right) \mu_{x}-\frac{1}{6} u_{x} \mu=0,
\end{aligned}
$$

where $\mu=\mu(x, t, k)$ and $k \in \mathbb{C}$ (note that the compatibility condition $\mu_{x x t}=\mu_{t x x}$ implies KdV for $u$ ). In Sobolev spaces, the KdV IVP has been studied by many authors using methods from partial differential equations and harmonic analysis. For instance, Kenig, Ponce, and Vega $^{34}$ proved local well-posedness in $H^{s}(\mathbb{R})$ for $s>-3 / 4$ by using Bourgain spaces, which had been introduced by Bourgain ${ }^{8}$ to establish well-posedness for $s=0$. Later, this was extended to a global well-posedness result by Colliander et al. ${ }^{12}$ Moreover, Christ, Colliander, and $\mathrm{TaO}^{11}$ proved that the KdV solution map fails to be uniformly continuous in $H^{s}$ for $s<-3 / 4$ (this result was first proved by Kenig, Ponce, and Vega ${ }^{36}$ for the complex-valued problem). The critical exponent $s=-3 / 4$ was settled by Guo, ${ }^{28}$ who proved global well-posedness of KdV in $H^{-3 / 4}(\mathbb{R})$. Prior to these results, Kenig, Ponce, and Vega ${ }^{33}$ had established well-posedness of $\operatorname{KdV}$ in $H^{s}(\mathbb{R})$ for $s>\frac{3}{4}$ by introducing an appropriate solution space. Some other earlier results can be found in the work of Bona and Smith, ${ }^{2}$ Saut and Temam, ${ }^{44}$ Constantin and Saut, ${ }^{14}$ Kenig, Ponce, and Vega, ${ }^{35}$ Bona and Saut, ${ }^{1}$ and the references therein.

In contrast to the IVP, the analysis of IBVPs for KdV [and other nonlinear evolution equations like the nonlinear Schrödinger (NLS) equation] is less developed. One of the difficulties arising in the IBVP setting was identified early on by noting that the Fourier transform, which is used in the Cauchy problem to solve the forced linear IVP and in turn defines the iteration map for showing well-posedness of the nonlinear IVP after replacing the forcing with the nonlinearity, is no longer available. In fact, the lack of Fourier transform led to two independent approaches that were introduced in the early 2000s for the well-posedness of the KdV equation on the half-line, namely, the studies of Colliander, Kenig, and Holmer, ${ }^{13,32}$ and of Bona, Sun, and Zhang. ${ }^{3,5}$ In the first approach, which has also been adapted for NLS on the half-line by Holmer, ${ }^{31}$ the forced linear IBVP is written as a superposition of IVPs on the line and the powerful Fourier analysis machinery is exploited. In the second approach, the forced linear IBVP is solved via a Laplace transform in the temporal variable.

As usual in contraction mapping schemes, the iteration map used in each of the above two approaches provides the basis for all of the subsequent well-posedness analysis. Hence, using an iteration map which is natural to the IBVP setting is crucial in optimizing this analysis. In this connection, we note that a novel approach has recently been introduced for proving well-posedness of IBVPs for nonlinear evolution equations. This approach has already been employed for the NLS, KdV, and "good" Boussinesq equations on the halfline $\mathrm{e}^{21,20,30}$ and overcomes the lack of the Fourier transform in the IBVP setting by exploiting the relevant solution formula produced via Fokas' unified transform method. ${ }^{17,19}$ The Fokas method can be used for solving linear evolution equations of arbitrary spatial order and dimension and supplemented with any type of admissible boundary data. In this light, it can be regarded as the analog of the Fourier transform in the case of linear IBVPs and hence comes forth as the natural way of defining the iteration map to be used for showing well-posedness of nonlinear IBVP via contraction mapping. The essence of the new approach lies in the analysis of the pure linear IBVP, i.e., of the case of zero initial data and zero forcing. In particular, estimating the Fokas solution formula of this problem reveals the correct space for the boundary data of the nonlinear problem. This step makes crucial use of the boundedness of the Laplace transform in $L^{2}$.

To state our results precisely, we recall the following spaces. For $s \in \mathbb{R}$ and $(a, b) \subset \mathbb{R}$, an interval that could extend to infinity on either side, the Sobolev space $H^{s}(a, b)$ is defined by

$$
H^{s}(a, b)=\left\{f: f=\left.F\right|_{(a, b)} \text { where } F \in H^{s}(\mathbb{R}) \text { and }\|f\|_{H^{s}(a, b)} \doteq \inf _{F \in H^{s}(\mathbb{R})}\|F\|_{H^{s}(\mathbb{R})}<\infty\right\}
$$

and the Sobolev norm on $\mathbb{R}$ is defined by

$$
\|F\|_{H^{s}(\mathbb{R})} \doteq\left(\int_{\xi \in \mathbb{R}}\left(1+\xi^{2}\right)^{2}|\widehat{F}(\xi)|^{2} d \xi\right)^{\frac{1}{2}}
$$

where $\widehat{F}(\xi)$ is the Fourier transform on $\mathbb{R}$ defined by

$$
\widehat{F}(\xi) \doteq \int_{\mathbb{R}} e^{-i x \xi} F(x) d x, \quad \xi \in \mathbb{R} .
$$


Furthermore, following Ref. 33, for $s \geqslant 0$, we define the Banach space

$$
X \doteq\left\{u \in C\left([0, T] ; H_{x}^{s}(0, \ell)\right):\|u\|_{X} \doteq \lambda^{T}(u)=\max \left\{\lambda_{1, s}^{T}(u), \lambda_{2, s}^{T}(u), \lambda_{3}^{T}(u)\right\}<\infty\right\},
$$

where the norms $\lambda_{1, s}^{T}(u), \lambda_{2, s}^{T}(u), \lambda_{3}^{T}(u)$ are defined by

$$
\begin{gathered}
\lambda_{1, s}^{T}(u) \doteq \sup _{t \in[0, T]}\|u(t)\|_{H_{x}^{s}(0, \ell)}, \\
\lambda_{2, s}^{T}(u) \doteq\left(\sup _{x \in[0, \ell]} \int_{0}^{T}\left|D_{x}^{s} \partial_{x} u(x, t)\right|^{2} d t\right)^{\frac{1}{2}}, \\
\lambda_{3}^{T}(u) \doteq\left(\int_{0}^{T} \sup _{x \in[0, \ell]}\left|\partial_{x} u(x, t)\right|^{4} d t\right)^{\frac{1}{4}}, \\
\left|\mathcal{D}_{x}^{s} u(x, t)\right|^{2} \doteq\left\{\begin{array}{c}
\left|\partial_{x}^{s} u(x, t)\right|^{2}, \quad s \in \mathbb{N} \doteq\{0,1,2, \cdots\} \\
\int_{0}^{\ell-x} \frac{\left|\partial_{x}^{\lfloor s\rfloor} u(x+z, t)-\partial_{x}^{\lfloor s\rfloor} u(x, t)\right|^{2}}{z^{1+2 \beta}} d z, \quad s=\lfloor s\rfloor+\beta, 0<\beta<1 .
\end{array}\right.
\end{gathered}
$$

Next, using the above definitions, we state our first result as follows.

Theorem 1.1 (Well-posedness of $\mathrm{KdV}$ on an interval). Let $\frac{3}{4}<s<1$. For data $u_{0} \in H_{x}^{s}(0, \ell), g_{0}, h_{0} \in H_{t}^{\frac{s+1}{3}}(0, T), h_{1} \in H_{t}^{\frac{s}{3}}(0, T)$, and satisfying the compatibility conditions

$$
u_{0}(0)=g_{0}(0), \quad u_{0}(\ell)=h_{0}(0)
$$

there exists $T^{*}, 0<T^{*} \leqslant T<1$ with

$$
T^{*}=\min \left\{T, \frac{1}{16^{3} c_{s}^{6}(1+\sqrt{\ell})^{3}\left\|\left(u_{0}, g_{0}, h_{0}, h_{1}\right)\right\|_{D}^{3}}\right\}, \quad c_{s}>0,
$$

where $\|\cdot\|_{D}$ is the data norm defined by

$$
\left\|\left(u_{0}, g_{0}, h_{0}, h_{1}\right)\right\|_{D}=\left\|u_{0}\right\|_{H_{x}^{s}(0, \ell)}+\left\|g_{0}\right\|_{H_{t}^{\frac{s+1}{3}}(0, T)}+\left\|h_{0}\right\|_{H_{t}^{\frac{s+1}{3}}(0, T)}+\left\|h_{1}\right\|_{H_{t}^{\frac{s}{3}}(0, T)}
$$

such that the KdV IBVP (1.1) has a unique solution $u$ in the Banach space

$$
X \doteq\left\{u \in C\left(\left[0, T^{*}\right] ; H_{x}^{s}(0, \ell)\right): \lambda^{T^{*}}(u)<\infty\right\}
$$

Also, the solution satisfies the size estimate

$$
\|u\|_{X} \leqslant 2 c_{s}\left\|\left(u_{0}, g_{0}, h_{0}, h_{1}\right)\right\|_{D} .
$$

Finally, the data to solution map $\left\{u_{0}, g_{0}, h_{0}, h_{1}\right\} \mapsto u$ is locally Lipschitz continuous.

The above theorem is a natural extension to an interval of the well-posedness result of the KdV IBVP on the half-line proved in Ref. 20 using a novel approach based on the Fokas method. For well-posedness results of the KdV IBVP on an interval using the other two approaches mentioned earlier, we refer the reader to Refs. 4 and 32.

The Proof of Theorem 1.1 is based on the solution formula of the associated forced linear KdV IBVP produced via the Fokas method. Replacing in this formula the forcing by the KdV nonlinearity gives an iteration map which we prove to be a contraction in the solution space $X$ defined by (1.15). Therefore, we begin with the following linear KdV IBVP with forcing: 


$$
\begin{aligned}
& \partial_{t} u+\partial_{x}^{3} u=f(x, t), \quad 0<x<\ell, 0<t<T, \\
& u(x, 0)=u_{0}(x) \in H_{x}^{s}(0, \ell), \\
& u(0, t)=g_{0}(t) \in H_{t}^{\frac{s+1}{3}}(0, T), \quad u(\ell, t)=h_{0}(t) \in H_{t}^{\frac{s+1}{3}}(0, T), \quad u_{x}(\ell, t)=h_{1}(t) \in H_{t}^{\frac{s}{3}}(0, T) .
\end{aligned}
$$

Using the Fokas method, also referred to in the literature as the unified transform method (UTM), we get the following solution to the problem (1.17):

$$
\begin{aligned}
& u(x, t)=S\left[u_{0}, g_{0}, h_{0}, h_{1} ; f\right](x, t) \\
\doteq & \frac{1}{2 \pi} \int_{-\infty}^{\infty} e^{i k x+i k^{3} t}\left[\hat{u}_{0}(k)+F(k, T)\right] d k+\frac{1}{2 \pi} \int_{\partial D^{+}} e^{i k x+i k^{3} t}\left\{\frac { 1 } { \Delta ( k ) } \left[N(k, T)\left[(\alpha+1) e^{-i \alpha k \ell}-e^{-i \alpha^{2} k \ell}\right]\right.\right. \\
& \left.\left.-\left[N(\alpha k, T)(\alpha+1)-N\left(\alpha^{2} k, T\right)\right] e^{-i k \ell}\right]+k^{2} \tilde{g}_{0}\left(k^{3}, t\right)\right\} d k+\frac{1}{2 \pi} \int_{\partial D_{1}^{-}} e^{i k(x-\ell)+i k^{3} t}\left\{\frac{1}{\Delta(k)}[\alpha N(k, T)\right. \\
& \left.\left.-(\alpha+1) N(\alpha k, T)+N\left(\alpha^{2} k, T\right)\right]+i k \tilde{h}_{1}\left(k^{3}, T\right)-k^{2} \tilde{h}_{0}\left(k^{3}, T\right)\right\} d k+\frac{1}{2 \pi} \int_{\partial D_{2}^{-}} e^{i k(x-\ell)+i k^{3} t} \\
& \cdot\left\{\frac{1}{\Delta(k)}\left[\alpha N(k, T)-(\alpha+1) N(\alpha k, T)+N\left(\alpha^{2} k, T\right)\right]+i k \tilde{h}_{1}\left(k^{3}, T\right)-k^{2} \tilde{h}_{0}\left(k^{3}, T\right)\right\} d k,
\end{aligned}
$$

where

$$
\begin{gathered}
\Delta(k)=e^{-i k \ell} \alpha-e^{-i \alpha k \ell}(\alpha+1)+e^{-i \alpha^{2} k \ell}, \quad \alpha=e^{\frac{2 \pi}{3} i}, \\
N(k, t)=\hat{u}_{0}(k)+F(k, t)-k^{2} \tilde{g}_{0}\left(k^{3}, t\right)-e^{-i k \ell}\left[i k \tilde{h}_{1}\left(k^{3}, t\right)-k^{2} \tilde{h}_{0}\left(k^{3}, t\right)\right], \\
F(k, t) \doteq \int_{0}^{t} e^{-i k^{3} \tau} \hat{f}(k, \tau) d \tau=\int_{0}^{t} e^{-i k^{3} \tau} \int_{0}^{\ell} e^{-i k x} f(x, \tau) d x d \tau, \\
\tilde{g}_{j}(k, t) \doteq \int_{0}^{t} e^{-i k \tau} \partial_{x}^{j} u(0, \tau) d \tau, \quad \tilde{h}_{j}(k, t) \doteq \int_{0}^{t} e^{-i k \tau} \partial_{x}^{j} u(\ell, \tau) d \tau .
\end{gathered}
$$

Theorem 1.2 (Forced linear KdV estimate on an interval). Suppose that $\frac{1}{2}<s<\frac{3}{2}$. Then the Fokas formula (1.18) defines a solution $u \in C\left([0, T] ; H_{x}^{s}(0, \ell)\right)$ to the forced linear KdV IBVP (1.17) with compatibility condition (1.12), which satisfies the estimate

$$
\begin{aligned}
\sup _{t \in[0, T]}\left\|S\left[u_{0}, g_{0}, h_{0}, h_{1} ; f\right](t)\right\|_{H_{x}^{s}(0, \ell)} \leqslant & c_{s}\left[\left\|u_{0}\right\|_{H_{x}^{s}(0, \ell)}+\left\|g_{0}\right\|_{H_{t}^{\frac{s+1}{3}}(0, T)}+\left\|h_{0}\right\|_{H_{t}^{\frac{s+1}{3}}(0, T)}+\left\|h_{1}\right\|_{H_{t}^{\frac{s}{3}}(0, T)}\right. \\
& \left.+\max \left\{T^{\frac{2-s}{3}}, T^{\frac{3-2 s}{3}}\right\}\left(\int_{0}^{T}\|f(t)\|_{H_{x}^{s}(0, \ell)}^{2} d t\right)^{\frac{1}{2}}\right] .
\end{aligned}
$$

The literature on $\mathrm{KdV}$ is vast. More results on $\mathrm{KdV}$, and in particular, about the IBVP on the half-line and an interval for the KdV and the cubic NLS equations that has been studied via the integrable nonlinear extension of the Fokas method, can be found in Refs. 7, 6, 9, 15, 16, $18,24,22,23,40,25,37,41,46$, and 47 and the references therein. Also, for a thorough introduction to the Fokas method, we refer the reader to the monograph ${ }^{19}$ and the review article. ${ }^{26}$ Finally, we mentioned that recently Özsar and Yolcu ${ }^{43}$ have used the unified transform method for proving global well-posedness results for the biharmonic nonlinear Schrödinger equation on the half-line.

Structure. The paper is organized as follows. In Sec. II, we prove Theorem 1.2 by decomposing the forced linear KdV IBVP (1.17) into two simpler problems, a forced IBVP on the half-line and a reduced IBVP on the interval with all data zero except for the boundary data at the right endpoint. In Sec. III, we prove the key estimate for the Fokas solution of the reduced IBVP (2.12), where the boundedness of the Laplace transform in $L^{2}$ plays a crucial role. In Sec. IV, we state all the estimates needed for the solution of the forced linear IBVP on half-line, which was used in the decomposition of our interval IBVP. Some of these estimates are taken from Ref. 20, and the rest are proved here. In Sec. V, we recall the iteration map defined by the Fokas solution formula when the forcing $f$ is replaced with the KdV nonlinearity and provide the bilinear estimate needed for estimating the quantity $\int_{0}^{T}\left\|u w_{x}(t)\right\|_{H_{x}^{s}(0, \ell)}^{2} d t$ in the solution space $X$. Also, we estimate the solution map in the $X$-norm [see estimate (5.12)]. Finally, in Sec. VI, we combine Theorem 1.2 and the results of Sec. V in order to prove Theorem 1.1 for the well-posedness of the KdV IBVP (1.1). We conclude with Sec. VII, where we provide an outline of the derivation of the solution formula to the forced linear KdV (1.17) via the Fokas method. 


\section{PROOF OF FORCED LINEAR KDV IBVP ESTIMATE (THEOREM 1.2)}

To prove Theorem 1.2, we decompose the forced linear KdV IBVP (1.17) into two simpler problems A and B. Problem A is the following forced IBVP on the half-line.

\section{Problem A: Half-line problem}

$$
\begin{array}{lr}
\partial_{t} w+\partial_{x}^{3} w=F(x, t) \in C\left([0, T] ; H_{x}^{s}(0, \infty)\right), & x \in(0, \infty), t \in(0, T), \\
w(x, 0)=w_{0}(x) \in H_{x}^{s}(0, \infty), & x \in[0, \infty), \\
w(0, t)=g_{0}(t) \in H_{t}^{\frac{s+1}{3}}(0, T), & t \in[0, T]
\end{array}
$$

where $w_{0}(x)$ and $F(x, t)$ are extensions to half-line of $u_{0}(x)$ and $f(x, t)$ from the interval $(0, \ell)$ satisfying the estimates

$$
\left\|w_{0}\right\|_{H^{s}(0, \infty)} \leqslant 2\left\|u_{0}\right\|_{H^{s}(0, \ell)}, \quad\|F\|_{C\left([0, T] ; H^{s}(0, \infty)\right)} \leqslant 2\|f\|_{C\left([0, T] ; H^{s}(0, \ell)\right)} .
$$

Using the Fokas method, we find that the solution to this half-line problem is given by

$$
\begin{aligned}
S\left[w_{0}, g_{0} ; F\right] & \doteq \frac{1}{2 \pi} \int_{-\infty}^{\infty} e^{i k x+i k^{3} t}\left[\widehat{w}_{0}(k)+F(k, t)\right] d k \\
& +\frac{1}{2 \pi} \int_{\partial D^{+}} e^{i k x+i k^{3} t}\left\{\alpha\left[\widehat{w}_{0}(\alpha k)+F(\alpha k, t)\right]+\alpha^{2}\left[\widehat{w}_{0}\left(\alpha^{2} k\right)+F\left(\alpha^{2} k, t\right)\right]\right\} d k \\
& -\frac{3}{2 \pi} \int_{\partial D^{+}} e^{i k x+i k^{3} t} k^{2} \tilde{g}_{0}\left(k^{3}, t\right) d k
\end{aligned}
$$

For this solution, we shall need the following linear estimates, which were proved in Ref. 20, except the last one that involves the spatial derivative of the solution.

Theorem 2.1 (Ref. 20). If $\frac{1}{2}<s<\frac{3}{2}$, then the Fokas method formula $w=S\left[w_{0}, g_{0} ; F\right]$ in $(2.3)$ defines a solution $w \in C\left([0, T] ; H_{x}^{s}(0, \infty)\right)$ to the linear $K d V I B V P(2.1)$ with the compatibility condition $w_{0}(0)=g_{0}(0)$ and satisfying the space estimate

$$
\sup _{t \in[0, T]}\|w(t)\|_{H_{x}^{s}(0, \infty)} \leqslant c_{s}\left(\left\|w_{0}\right\|_{H_{x}^{s}(0, \infty)}+\left\|g_{0}\right\|_{H_{t}^{\frac{s+1}{3}}(0, T)}+T^{\frac{2-s}{3}}\|F\|_{L^{2}\left([0, T] ; H_{x}^{s}(0, \infty)\right)}\right),
$$

the time estimate for the solution

$$
\sup _{x \in[0, \infty)}\|w(x)\|_{H_{t}^{\frac{s+1}{3}}(0, T)} \leqslant c_{s}\left(\left\|w_{0}\right\|_{H_{x}^{s}(0, \infty)}+\left\|g_{0}\right\|_{H_{t}^{\frac{s+1}{3}}(0, T)}+T^{\frac{2-s}{3}}\|F\|_{L^{2}\left([0, T] ; H_{x}^{s}(0, \infty)\right)}\right)
$$

and also the time estimate for the derivative of the solution

$$
\begin{aligned}
\sup _{x \in[0, \infty)}\left\|w_{x}(x)\right\|_{H_{t}^{\frac{s}{3}}(0, T)} \leqslant & c_{s}\left(\left\|w_{0}\right\|_{H_{x}^{s}(0, \infty)}+\left\|g_{0}\right\|_{H_{t}^{\frac{s+1}{3}}(0, T)}\right. \\
& \left.+\max \left\{T^{\frac{2-s}{3}}, T^{\frac{3-2 s}{3}}\right\}\|F\|_{L^{2}\left([0, T] ; H_{x}^{s}(0, \infty)\right)}\right) .
\end{aligned}
$$

Thanks to the above estimates and using linearity, we can subtract problem A from forced linear KdV IBVP (1.17) on the interval and obtain the following simpler pure IBVP.

Problem B: Pure IBVP on the interval

$$
\begin{aligned}
& \partial_{t} v+\partial_{x}^{3} v=0, \quad x \in(0, \ell), t \in(0, T), \\
& v(x, 0)=0, \quad x \in(0, \ell), \\
& v(0, t)=0, \quad t \in(0, T), \\
& v(\ell, t)=h_{0}(t)-w(\ell, t) \doteq h_{0}^{*}(t) \in H_{t}^{\frac{s+1}{3}}(0, T), v_{x}(\ell, t)=h_{1}(t)-w_{x}(\ell, t) \doteq h_{1}^{*}(t) \in H_{t}^{\frac{s}{3}}(0, T) .
\end{aligned}
$$

Next, we shall extend the reduced boundary data $h_{0}^{*}(t)$ and $h_{1}^{*}(t)$ appropriately so that they have compact support, in which case the time transform reads as Fourier transform. For this, first we extend them to the whole line as Sobolev functions of the same class, and then we 
multiply them by a test function which is equal to one on $[0, T]$ and supported in $[-2,2]$. Finally, keeping the same notation, we apply the following lemma which can be found in Ref. 42 .

Lemma 2.1. For a general function $h^{*}(t) \in H_{t}^{s}(0,2), s \geqslant 0$, let

$$
\tilde{h}^{*}(t) \doteq\left\{\begin{array}{lr}
h^{*}(t), & t \in(0,2) \\
0, & \text { elsewhere }
\end{array}\right.
$$

If $0 \leqslant s<\frac{1}{2}$, then for some $c_{s}>0$, we have

$$
\left\|\tilde{h}^{*}\right\|_{H_{t}^{s}(\mathbb{R})} \leqslant c_{s}\left\|h^{*}\right\|_{H_{t}^{s}(0,2)} .
$$

If $\frac{1}{2}<s \leqslant 1$, then for estimate (2.8) to hold, we must have the condition $h^{*}(0)=h^{*}(2)=0$.

For $h_{1}^{*}(t)$, which belong to a Sobolev space with the exponent in $\left(\frac{1}{6}, \frac{1}{2}\right)$, applying the first part of the lemma, while for $h_{0}^{*}(t)$, which belong to a Sobolev space with the exponent in $\left(\frac{1}{2}, \frac{5}{6}\right)$, applying the second part of the lemma, we have

$$
\left\|\tilde{h}_{0}^{*}\right\|_{H_{t}^{\frac{s+1}{3}}(\mathbb{R})} \lesssim\left\|h_{0}^{*}\right\|_{H_{t}^{\frac{s+1}{3}}(0, T)}, \quad\left\|\tilde{h}_{1}^{*}\right\|_{H_{t}^{\frac{5}{3}}(\mathbb{R})} \lesssim\left\|h_{1}^{*}\right\|_{H_{t}^{\frac{s}{3}}(0, T)} .
$$

Now, letting

$$
h_{2} \doteq \tilde{h}_{0}^{*} \quad \text { and } \quad h_{3} \doteq \tilde{h}_{1}^{*} \text {, }
$$

and using the time estimate (2.5) and the estimate for the derivative (2.6), we get

$$
\begin{aligned}
\left\|h_{2}\right\|_{H_{t}^{\frac{s+1}{3}}(0,2)} \lesssim\left\|w_{0}\right\|_{H_{x}^{s}(0, \infty)} & +\left\|g_{0}\right\|_{H_{t}^{\frac{s+1}{3}}(0, T)}+\left\|h_{0}\right\|_{H_{t}^{\frac{s+1}{3}}(0, T)}+T^{\frac{2-s}{3}}\|F\|_{L^{2}\left([0, T] ; H_{x}^{s}(0, \infty)\right)}, \\
\left\|h_{3}\right\|_{H_{t}^{\frac{s}{3}}(0,2)} \lesssim\left\|w_{0}\right\|_{H_{x}^{s}(0, \infty)} & +\left\|g_{0}\right\|_{H_{t}^{\frac{s+1}{3}}(0, T)}+\left\|h_{1}\right\|_{H_{t}^{\frac{s}{3}}(0, T)} \\
& +\max \left\{T^{\frac{2-s}{3}}, T^{\frac{3-2 s}{3}}\right\}\|F\|_{L^{2}\left([0, T] ; H_{x}^{s}(0, \infty)\right)}
\end{aligned}
$$

Reduced pure IBVP on interval. Thus, we have reduced IBVP (2.7) to the following simpler IBVP with boundary data $h_{0}^{*}$ and $h_{1}^{*}$ extended nicely to $h_{2}$ and $h_{3}$ so that they are compactly supported (allowing us to replace time transform with Fourier transform, which is used in Sobolev norms)

$$
\begin{aligned}
& \partial_{t} v+\partial_{x}^{3} v=0, \quad x \in(0, \ell), t \in(0,2), \\
& v(x, 0)=0, \quad x \in(0, \ell) \\
& v(0, t)=0, \quad t \in(0,2), \\
& v(\ell, t)=h_{2}(t) \in H_{t}^{\frac{s+1}{3}}(0,2), \quad v_{x}(\ell, t)=h_{3}(t) \in H_{t}^{\frac{s}{3}}(0,2) .
\end{aligned}
$$

Applying the Fokas solution formula (1.18) with $f=u_{0}=g_{0}=0$ and using the fact that now $h_{2}$ and $h_{3}$ are compactly supported in the interval $[0,2]$, which gives

$$
\tilde{h}_{2}(k, 2)=\int_{0}^{2} e^{-i k \tau} h_{2}(\tau) d \tau=\int_{\mathbb{R}} e^{-i k \tau} h_{2}(\tau) d \tau=\hat{h}_{2}(k), \quad \text { and } \quad \tilde{h}_{3}(k, 2)=\hat{h}_{3}(k),
$$

we obtain the following formula for the solution to the reduced IBVP (2.12):

$$
\begin{aligned}
v(x, t)= & S\left[0,0, h_{2}, h_{3} ; 0\right] \doteq-\frac{1}{2 \pi} \int_{\partial D^{+} \cup \partial D_{1}^{-} \cup \partial D_{2}^{-}} \frac{e^{i k(x-\ell)+i k^{3} t}}{\Delta(k)}\left(1-\alpha^{2}\right)\left(e^{-i \alpha k \ell}-e^{-i \alpha^{2} k \ell}\right) i k \hat{h}_{3}\left(k^{3}\right) d k \\
& +\frac{1}{2 \pi} \int_{\partial D^{+} \cup \partial D_{1}^{-} \cup \partial D_{2}^{-}} \frac{e^{i k(x-\ell)+i k^{3} t}}{\Delta(k)}\left[\left(\alpha-\alpha^{2}\right) e^{-i \alpha k \ell}-(1-\alpha) e^{-i \alpha^{2} k \ell}\right] k^{2} \hat{h}_{2}\left(k^{3}\right) d k,
\end{aligned}
$$

where $D_{1}^{+}, D_{1}^{-}, D_{2}^{-}$are shown in Fig. 1. Now, we are ready to state the key estimate for the reduced KdV IBVP, which plays a crucial role in our approach. More precisely, we have the following result, whose proof is given in Sec. III. 


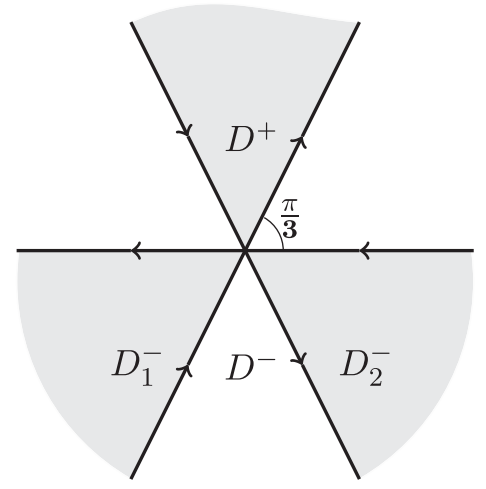

FIG. 1. The regions $D^{+}$and $D_{1}^{-}, D_{2}^{-}$in the complex k-plane.

Theorem 2.2. If $0 \leqslant s<\frac{3}{2}$, then the UTM formula (2.13) defines a solution $v \in C\left([0,2] ; H_{x}^{s}(0, \infty)\right)$ to the reduced IBVP (2.12) which satisfies the space estimate

$$
\sup _{t \in[0,2]}\left\|S\left[0,0, h_{2}, h_{3} ; 0\right]\right\|_{H_{x}^{s}(0, \ell)} \leqslant c_{s}\left(\left\|h_{2}\right\|_{H_{t}^{\frac{s+1}{3}}(0,2)}+\left\|h_{3}\right\|_{H_{t}^{\frac{5}{3}}(0,2)}\right)
$$

where $c_{s}>0$ is a constant depending on $s$.

Next, combining the above estimate with the half-line estimate (2.4), we prove Theorem 1.2.

Proof of Theorem 1.2. Taking advantage of our decomposition of the solution to the forced linear KdV IBVP, we have

$$
u=S\left[u_{0}, g_{0}, h_{0}, h_{1} ; f\right]=w+v=S\left[w_{0}, g_{0} ; F\right]+S\left[0,0, h_{2}, h_{3} ; 0\right], \quad x \in(0, \ell), t \in(0, T)
$$

Therefore,

$$
\sup _{t \in[0, T]}\|u\|_{H_{x}^{s}(0, \ell)} \leqslant \sup _{t \in[0, T]}\left\|S\left[w_{0}, g_{0} ; F\right]\right\|_{H_{x}^{s}(0, \infty)}+\sup _{t \in[0,2]}\left\|S\left[0,0, h_{2}, h_{3} ; 0\right]\right\|_{H_{x}^{s}(0, \ell)}
$$

By Theorem 2.1, we have

$$
\begin{aligned}
\sup _{t \in[0, T]}\left\|S\left[w_{0}, g_{0} ; F\right]\right\|_{H_{x}^{s}(0, \infty)} & \leqslant c_{s}\left(\left\|w_{0}\right\|_{H_{x}^{s}(0, \infty)}+\left\|g_{0}\right\|_{H_{t}^{\frac{s+1}{3}}(0, T)}+T^{\frac{3-2 s}{3}}\|F\|_{L^{2}\left([0, T] ; H_{x}^{s}(0, \infty)\right)}\right) \\
& \leqslant 2 c_{s}\left(\left\|u_{0}\right\|_{H_{x}^{s}(0, \infty)}+\left\|g_{0}\right\|_{H_{t}^{\frac{s+1}{3}}(0, T)}+T^{\frac{3-2 s}{3}}\|f\|_{L^{2}\left([0, T] ; H_{x}^{s}(0, \ell)\right)}\right),
\end{aligned}
$$

and by Theorem 2.2, we get

$$
\begin{aligned}
\sup _{t \in[0,2]}\left\|S\left[0,0, h_{2}, h_{3} ; 0\right]\right\|_{H_{x}^{s}(0, \ell)} & \leqslant c_{s}\left(\left\|h_{2}\right\|_{H_{t}^{\frac{s+1}{3}}(0,2)}+\left\|h_{3}\right\|_{H_{t}^{\frac{s}{3}}(0,2)}\right) \\
& \leqslant c_{s}\left(\left\|u_{0}\right\|_{H_{x}^{s}(0, \infty)}+\left\|g_{0}\right\|_{H_{t}^{\frac{s+1}{3}}(0, T)}+\left\|h_{0}\right\|_{H_{t}^{\frac{s+1}{3}}(0, T)}+\left\|h_{1}\right\|_{H_{t}^{\frac{s}{3}}(0, T)}\right. \\
& \left.+\max \left\{T^{\frac{2-s}{3}}, T^{\frac{3-2 s}{3}}\right\}\|f\|_{L^{2}\left([0, T] ; H_{x}^{s}(0, \ell)\right)}\right) .
\end{aligned}
$$

Putting these together, we obtain the desired estimate (1.23). 


\section{PROOF OF ESTIMATE FOR REDUCED IBVP (THEOREM 2.2)}

We begin by writing the solution (2.13) to the reduced IBVP as follows:

$$
v=v_{1}+v_{2}+v_{3}
$$

where $v_{1}$ is the part of $v$ over $\partial D^{+}, v_{2}$ is the part of $v$ over $\partial D_{1}^{-}$, and $v_{3}$ is the part of $v$ over $\partial D_{2}^{-}$(see Fig. 1). Here, we shall estimate only $v_{1}$ since the rest are similar. To do this, we further decompose $v_{1}$ into the parts $v_{11}$ involving $h_{3}$ and $v_{12}$ involving $h_{2}$, that is, $v_{1}=v_{11}+v_{12}$, where

$$
\begin{aligned}
& v_{11} \doteq-\frac{1}{2 \pi} \int_{\partial D^{+}} \frac{e^{i k(x-\ell)+i k^{3} t}}{\Delta(k)}\left(1-\alpha^{2}\right)\left(e^{-i \alpha k \ell}-e^{-i \alpha^{2} k \ell}\right) i k \hat{h}_{3}\left(k^{3}\right) d k \\
& v_{12} \doteq \frac{1}{2 \pi} \int_{\partial D^{+}} \frac{e^{i k(x-\ell)+i k^{3} t}}{\Delta(k)}\left[\left(\alpha-\alpha^{2}\right) e^{-i \alpha k \ell}-(1-\alpha) e^{-i \alpha^{2} k \ell}\right] k^{2} \hat{h}_{2}\left(k^{3}\right) d k .
\end{aligned}
$$

In fact, here, we estimate only $v_{11}$. The estimation of $v_{12}$ is similar.

Space estimate for $\boldsymbol{v}_{11}$. We rewrite $v_{11}$ as

$$
v_{11} \simeq \int_{\partial D^{+}} C(k) e^{i k x+i k^{3} t} k \hat{h}_{3}\left(k^{3}\right) d k
$$

where

$$
C(k)=\frac{e^{-i k \ell} \cdot\left(e^{-i \alpha k \ell}-e^{-i \alpha^{2} k \ell}\right)}{e^{-i k \ell} \alpha-e^{-i \alpha k \ell}(\alpha+1)+e^{-i \alpha^{2} k \ell}}, \quad k \in \partial D^{+} .
$$

First, we observe that the denominator in (3.4), which in (1.19) is denoted by $\Delta(k)$, has no zeros on $\partial D^{+}$for $k \neq 0$ (see Lemma 7.1). Also, we note that when $k$ is on $\partial D^{+}$then $\alpha k$ is on $\partial D_{1}^{-}$and $\alpha^{2} k$ is on $\partial D_{2}^{-}$. Hence, in $C(k)$, the exponential $\left|e^{-i k \ell}\right| \rightarrow \infty$ when $k \in \partial D^{+}$and $|k| \rightarrow \infty$, while the other exponentials stay bounded. This implies that

$$
|C(k)| \lesssim 1 \quad \text { as }|k| \longrightarrow \infty
$$

Moreover, when $k$ is near 0 , we see that the denominator $\Delta(k) \simeq k^{2}$ and the numerator is like $k$. Therefore, we have

$$
C(k) \simeq \frac{1}{k} \quad \text { as } \quad k \longrightarrow 0 .
$$

These suggest the splitting of the integral (3.3) into three terms, one near zero and the other two far away on $C_{1}$ and $C_{2}$, that is,

$$
v_{11} \simeq V_{0}+V_{1}+V_{2}
$$

where

$$
V_{j}(x, t)=\int_{C_{j}} C(k) e^{i k x+i k^{3} t} k \hat{h}_{3}\left(k^{3}\right) d k
$$

and $C_{j}, j=0,1,2$, are shown in Fig. 2. By the symmetry of the domain $D^{+}$, the estimate for $V_{1}$ is similar to the estimate for $V_{2}$. So, here, we derive the estimates only for $V_{2}$ and $V_{0}$.

Estimate of $V_{\mathbf{2}}$. Our method of estimation on the contour $C_{2}$ is similar to the one used in Ref. 20, where the key ingredient is the $L^{2}$ boundedness of the Laplace transform. Here, we reduce into a Laplace transform situation by using the parameterization $[1, \infty) \ni k \rightarrow a k \in C_{2}$ so that

$$
V_{2}(x, t)=\int_{1}^{\infty} C(a k) e^{i a k x-i k^{3} t}(a k) \hat{h}_{3}\left(-k^{3}\right) d k
$$

where $a=e^{\frac{\pi}{3} i}$, which gives $a^{3}=-1$. To prove the desired estimate for $V_{2}(x, t)$, we begin by estimating the Sobolev norm of $V_{2}(x, t)$, that is, $\left\|V_{2}(t)\right\|_{H_{x}^{s}(0, \ell)}$. For this, we recall that for any function $f(x)$ on $(0, \ell)$ the description of its $H^{s}(0, \ell)$-Sobolev norm in the physical space for $s \geqslant$ 0 is given by

$$
\|f\|_{H_{x}^{s}(0, \ell)}^{2}=\sum_{j=0}^{\lfloor s\rfloor}\left\|\partial_{x}^{j} f\right\|_{L^{2}(0, \ell)}^{2}+\left\|\partial_{x}^{\lfloor s\rfloor} f\right\|_{\beta}^{2},
$$




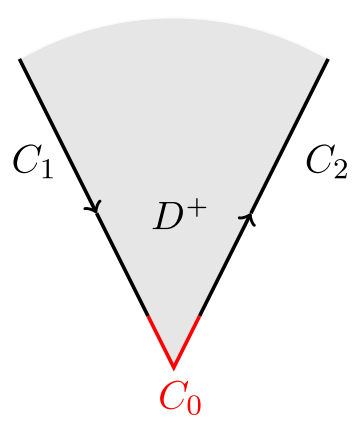

FIG. 2. Contours $C_{0}, C_{1}, C_{2}$.

where $s=\lfloor s\rfloor+\beta, \beta \in(0,1)$, with $s=\lfloor s\rfloor$ denoting the integer part of $s$. Furthermore, for any test function $\varphi$ defined on $(0, \ell)$ and $\beta \in(0,1)$, its fractional norm $\|\varphi\|_{\beta}$ is given by

$$
\|\varphi\|_{\beta}^{2} \doteq \int_{x_{2}=0}^{\ell} \int_{x_{1}=0}^{\ell} \frac{\left|\varphi\left(x_{1}\right)-\varphi\left(x_{2}\right)\right|^{2}}{\left|x_{1}-x_{2}\right|^{1+2 \beta}} d x_{1} d x_{2}=2 \int_{y=0}^{\ell} \int_{z=0}^{\ell-y} \frac{|\varphi(z+y)-\varphi(y)|^{2}}{z^{1+2 \beta}} d z d y,
$$

where the second equality is obtained by changing variables $x_{1}=z+y$ and $x_{2}=y$. So, in our case that we have $0 \leqslant s<3 / 2$, we need to estimate the $L^{2}$ norms of $V_{2}(t), \partial_{x} V_{2}(t)$ and the fractional norms of $V_{2}(t), \partial_{x} V_{2}(t)$.

Fractional norm of $\partial_{x}^{\lfloor s\rfloor} V_{2}$. Differentiating $j$ times $V_{2}(x, t)$ with respect to $x$, we obtain

$$
\partial_{x}^{j} V_{2}(x, t) \simeq \int_{1}^{\infty} C(a k) e^{i a k x-i k^{3} t} k^{j+1} \hat{h}_{3}\left(-k^{3}\right) d k
$$

For $j=\lfloor s\rfloor$ and $\beta \in(0,1)$, using the second form of fractional norm (3.10), we have

$$
\left\|\partial_{x}^{\lfloor s\rfloor} V_{2}(t)\right\|_{\beta}^{2} \simeq \int_{0}^{\ell} \int_{0}^{\ell-y} \frac{\left|\partial_{x}^{\lfloor s\rfloor} V_{2}(z+y, t)-\partial_{x}^{\lfloor s\rfloor} V_{2}(y, t)\right|^{2}}{|z|^{1+2 \beta}} d z d y \simeq \int_{0}^{\ell} \int_{0}^{\ell-y} \frac{\left|\int_{1}^{\infty}\right| e^{i a k(z+y)}-e^{i a k y}\left|G_{h}(k) d k\right|^{2}}{|z|^{1+2 \beta}} d z d y,
$$

where

$$
G_{h}(k)=C(a k) \cdot k^{\lfloor s\rfloor+1} e^{-i k^{3} t} \hat{h}_{3}\left(-k^{3}\right)
$$

The following crucial lemma, which is proved in Ref. 20, provides a way of handling the complex exponentials involved in the above integral.

Lemma 3.1 (Estimating along the complex UTM contours). For $\gamma=\gamma_{R}+i \gamma_{I}, \gamma_{I}>0$, we have

$$
\left|e^{i \gamma k x}-e^{i \gamma k y}\right| \leqslant \sqrt{2}\left(1+\frac{\left|\gamma_{R}\right|}{\gamma_{I}}\right)\left|e^{-\gamma_{I} k x}-e^{-\gamma_{I} k y}\right| \quad \forall x, y \geqslant 0 .
$$

Applying this lemma with $\gamma=e^{\frac{i \pi}{3}}=a$, which has imaginary part $a_{I}=\frac{\sqrt{3}}{2}>0$, we have

$$
\left|e^{i a k(z+y)}-e^{i a k y}\right| \leqslant c \cdot\left|e^{-a_{1} k(y+z)}-e^{-a_{1} k y}\right|
$$

Furthermore, making the change of variables $k^{\prime}=\frac{\sqrt{3}}{2} k$, replacing the upper limits $\ell$ and $\ell-y$ by $\infty$, and switching $d z$ with $d y$ integration, we get 


$$
\left\|\partial_{x}^{\lfloor s\rfloor} V_{2}(t)\right\|_{\beta}^{2} \lesssim \int_{z=0}^{\infty} \frac{1}{|z|^{\mid+2 \beta}} \int_{y=0}^{\infty}\left|\int_{k^{\prime}=\frac{\sqrt{3}}{2}}^{\infty} e^{-k^{\prime} y}\left(1-e^{-k^{\prime} z}\right) G_{h}\left(k^{\prime}, t\right) d k^{\prime}\right|^{2} d y d z
$$

Now, we need the following important result, whose proof follows from the one in Ref. 21 (see also the work of Hardy ${ }^{29}$ ), by multiplying $Q(k)$ by the characteristic function $\chi(c, \infty)(k)$.

Lemma 3.2 ( $L^{2}$ boundedness of the Laplace transform). Suppose that $Q(k) \in L_{k}^{2}(c, \infty), c \geqslant 0$. Then, the map $Q(k) \longmapsto \int_{c}^{\infty} e^{-k y} Q(k) d k$ is bounded from $L_{k}^{2}(c, \infty)$ into $L_{y}^{2}(0, \infty)$ with

$$
\left\|\int_{c}^{\infty} e^{-k y} Q(k) d k\right\|_{L_{y}^{2}(0, \infty)} \leqslant \sqrt{\pi}\|Q(k)\|_{L_{k}^{2}(c, \infty)} .
$$

Applying Lemma 3.2 with $Q\left(k^{\prime}\right)=\left(1-e^{-k^{\prime} z}\right) G_{h}\left(k^{\prime}, t\right)$ and switching $d z$ and $d k^{\prime}$, we have

$$
\left\|\partial_{x}^{\lfloor s\rfloor} V_{2}(t)\right\|_{\beta}^{2} \simeq \int_{k^{\prime}=\frac{\sqrt{3}}{2}}^{\infty}\left|G_{h}\left(k^{\prime}, t\right)\right|^{2} \int_{z=0}^{\infty} \frac{\left(1-e^{-k^{\prime} z}\right)^{2}}{|z|^{1+2 \beta}} d z d k^{\prime} .
$$

Finally, using the elementary estimate

$$
\int_{z=0}^{\infty} \frac{\left(1-e^{-k z}\right)^{2}}{|z|^{1+2 \beta}} d z \simeq|k|^{2 \beta}, \quad 0<\beta<1,
$$

we obtain

$$
\left\|\partial_{x}^{\lfloor s\rfloor} V_{2}(t)\right\|_{\beta}^{2} \simeq \int_{k^{\prime}=\frac{\sqrt{3}}{2}}^{\infty}\left|G_{h}\left(k^{\prime}, t\right)\right|^{2}\left|k^{\prime}\right|^{2 \beta} d k^{\prime} \lesssim \int_{k=1}^{\infty}\left|k^{\lfloor s\rfloor+1} \hat{h}_{3}\left(-k^{3}\right)\right|^{2}(k)^{2 \beta} d k \leqslant\left\|h_{3}\right\|_{H_{t}^{\frac{s}{3}}(\mathbb{R})} .
$$

$\boldsymbol{L}^{2}$ norm of $\partial_{x}^{j} V_{2}$. For $0 \leq j \leq\lfloor s\rfloor \in \mathbb{N}$, using (3.11) and changing variables as before, we obtain

$$
\left\|\partial_{x}^{j} V_{2}(t)\right\|_{L_{x}^{2}(0, \ell)}^{2} \lesssim\left\|\int_{a_{I}}^{\infty} e^{-k x}\left|k^{j+1} \hat{h}_{3}\left(-k^{3}\right)\right| d k\right\|_{L_{x}^{2}(0, \ell)}^{2} .
$$

Thus, applying Lemma 3.2 with $Q(k)=\left|k^{j+1} \hat{h}_{3}\left(-k^{3}\right)\right|$, we get

$$
\left\|\partial_{x}^{j} V_{2}(t)\right\|_{L_{x}^{2}(0, \ell)}^{2} \lesssim\left\|\left.k\right|^{j} \cdot k \hat{h}_{3}\left(-k^{3}\right)\right\|_{L_{k}^{2}\left(a_{I}, \infty\right)}^{2} \lesssim\left\|h_{3}\right\|_{H_{t}^{\frac{j}{3}}(\mathbb{R})} \leqslant\left\|h_{3}\right\|_{H_{t}^{\frac{5}{3}}(\mathbb{R})} .
$$

Finally, combining (3.9), (3.16), and (3.17), we obtain the desired estimate (2.14) for $V_{2}(x, t)$.

Estimate for $V_{0}$. Recall that $V_{0}$ is defined by integrating over the two symmetric segments in the complex $k$-plane starting at 0 and ending at $-\frac{1}{2}+\frac{\sqrt{3}}{2} i$ and the other at $\frac{1}{2}+\frac{\sqrt{3}}{2} i$, that is,

$$
V_{0}(x, t)=\frac{\left(1-\alpha^{2}\right) i}{2 \pi}\left\{\int_{-\frac{1}{2}+\frac{\sqrt{3}}{2} i}^{0+0 \cdot i} C(k) e^{i k x+i k^{3} t} k \hat{h}_{3}\left(k^{3}\right) d k+\int_{0+0 \cdot i}^{\frac{1}{2}+\frac{\sqrt{3}}{2} i} C(k) e^{i k x+i k^{3} t} k \hat{h}_{3}\left(k^{3}\right) d k\right\} .
$$

Here, we only estimate the second integral

$$
V_{0,2}(x, t) \doteq \int_{0+0 \cdot i}^{0.5+\frac{\sqrt{3}}{2} i} C(k) e^{i k x+i k^{3} t} k \hat{h}_{3}\left(k^{3}\right) d k
$$

since the estimation of the first one is similar. For any $j \in \mathbb{N}$, we have

$$
\partial_{x}^{j} V_{0,2}(x, t)=\int_{0+0 \cdot i}^{0.5+\frac{\sqrt{3}}{2} i}(C(k) k) \cdot e^{i k x+i k^{3} t}(i k)^{j} \hat{h}_{3}\left(k^{3}\right) d k
$$

Since for $k \in\left[0, \frac{1}{2}+\frac{\sqrt{3}}{2} i\right]$ and $x \in[0, \ell]$ we have $(C(k) k) \cdot e^{i k x+i k^{3} t}(i k)^{j}$ uniformly bounded, and 


$$
\left|\hat{h}_{3}\left(k^{3}\right)\right|=\left|\int_{0}^{2} e^{-i k^{3} t} h_{3}(t) d t\right| \lesssim\left\|h_{3}\right\|_{L^{1}(0,2)} \lesssim\left\|h_{3}\right\|_{L^{2}(0,2)},
$$

we see that $\left\|\partial_{x}^{j} V_{0,2}(t)\right\|_{L_{x}^{2}(0, \ell)} \lesssim\left\|h_{3}\right\|_{L^{2}(0,2)}$, which implies

$$
\left\|V_{0,2}(t)\right\|_{H_{x}^{u}(0, \ell)}=\sum_{j=1}^{\mu}\left\|\partial_{x}^{j} V_{0}(t)\right\|_{L_{x}^{2}(0, \ell)} \lesssim\left\|h_{3}\right\|_{L_{t}^{2}(0,2)} \lesssim\left\|h_{3}\right\|_{H_{t}^{\frac{s}{3}}(0,2)}
$$

for any positive integer $\mu$. Therefore, applying the last estimate for $\mu=\lfloor s\rfloor+1, s>0$, we get

$$
\left\|V_{0,2}(t)\right\|_{H_{x}^{s}(0, \ell)} \lesssim\left\|V_{0,2}(t)\right\|_{H_{x}^{(s)+1}(0, \ell)} \lesssim\left\|h_{3}\right\|_{H_{t}^{\frac{5}{3}}(0,2)} \leqslant\left\|h_{3}\right\|_{H_{t}^{\frac{5}{3}}(\mathbb{R})^{\prime}}
$$

which is the desired estimate (2.14) for $V_{0,2}(x, t)$. This completes the Proof of Theorem 2.2.

\section{PROOF OF FORCED LINEAR IBVP ESTIMATES ON HALF-LINE (THEOREM 2.1)}

To prove Theorem 2.1, we decompose the half-line IBVP (2.1) into simpler problems, and at the same time, we provide the basic estimates for each one of them. Then, using these, we prove the estimates contained in Theorem 2.1. In fact, here, we focus only on the derivation of the time estimate for the solution (2.5) and the time estimate for the derivative of the solution (2.6). The other estimates are contained in Ref. 20.

We begin by decomposing the forced linear IBVP (2.1) into a homogeneous problem (A) and an inhomogeneous problem (B). Then, we decompose both problems further in a convenient way.

\section{A. The homogeneous linear IBVP}

$$
\begin{array}{ll}
u_{t}+u_{x x x}=0, & x \in(0, \infty), t \in(0, T), \\
u(x, 0)=w_{0}(x) \in H_{x}^{s}(0, \infty), & x \in[0, \infty), \\
u(0, t)=g_{0}(t) \in H_{t}^{\frac{s+1}{3}}(0, T), & t \in[0, T],
\end{array}
$$

with the solution given by $u(x, t) \doteq S\left[w_{0}, g_{0} ; 0\right](x, t)$, which is defined in (2.3); it can be decomposed further into the following two problems: $\mathrm{A}_{1}$. The homogeneous linear initial value problem (IVP)

$$
\begin{array}{ll}
U_{t}+U_{x x x}=0, & x \in \mathbb{R}, t \in(0, T), \\
U(x, 0)=U_{0}(x) \in H_{x}^{s}(\mathbb{R}), & x \in \mathbb{R},
\end{array}
$$

where $U_{0} \in H_{x}^{s}(\mathbb{R})$ is an extension of the initial datum $w_{0} \in H_{x}^{s}(0, \infty)$ such that $\left\|U_{0}\right\|_{H_{x}^{s}(\mathbb{R})} \leqslant 2\left\|w_{0}\right\|_{H_{x}^{s}(0, \infty)}$ with the solution given by

$$
U(x, t)=S\left[U_{0} ; 0\right](x, t)=\frac{1}{2 \pi} \int_{\xi \in \mathbb{R}} e^{i \xi x+i \xi^{3} t} \widehat{U}_{0}(\xi) d \xi,
$$

where $\widehat{U}_{0}(\xi)=\int_{x \in \mathbb{R}} e^{-i \xi x} U_{0}(x) d x, \quad \xi \in \mathbb{R}$.

$\mathrm{A}_{2}$. The homogeneous linear IBVP with zero initial datum

$$
\begin{array}{ll}
u_{t}+u_{x x x}=0, & x \in(0, \infty), t \in(0, T), \\
u(x, 0)=0, & x \in[0, \infty), \\
u(0, t)=G_{0}(t) \doteq g_{0}(t)-U(0, t), & t \in[0, T],
\end{array}
$$

with solution $u(x, t) \doteq S\left[0, G_{0} ; 0\right](x, t)$, which is defined in (2.3).

\section{B. The forced linear IBVP with zero data}

$$
\begin{array}{ll}
u_{t}+u_{x x x}=F(x, t) \in C\left([0, T] ; H_{x}^{s}(0, \infty)\right), & x \in(0, \infty), t \in(0, T), \\
u(x, 0)=0, & x \in[0, \infty), \\
u(0, t)=0, & t \in[0, T],
\end{array}
$$


whose solution is $u(x, t) \doteq S[0,0 ; F](x, t)$, which is defined in (2.3). This problem can be further decomposed into the following two problems: $B_{1}$. The forced linear IVP with zero initial condition

$$
\begin{array}{ll}
W_{t}+W_{x x x}=F_{e}(x, t) \in C\left([0, T] ; H_{x}^{s}(\mathbb{R})\right), & x \in \mathbb{R}, t \in(0, T), \\
W(x, 0)=0, & x \in \mathbb{R},
\end{array}
$$

where $F_{e} \in C\left([0, T] ; H_{x}^{s}(\mathbb{R})\right)$ is an extension of the forcing $F \in C\left([0, T] ; H_{x}^{s}(0, \infty)\right)$ such that $\sup _{t \in[0, T]}\left\|F_{e}(t)\right\|_{H_{x}^{s}(\mathbb{R})} \leqslant 2 \sup _{t \in[0, T]}\|F(t)\|_{H_{x}^{s}(0, \infty)}$. The solution of this problem is given by the Duhamel representation

$$
\begin{aligned}
W(x, t) \doteq S\left[0 ; F_{e}\right](x, t) & =-\frac{i}{2 \pi} \int_{\xi \in \mathbb{R}} \int_{t^{\prime}=0}^{t} e^{i \xi x+i \xi^{\xi}\left(t-t^{\prime}\right)} \widehat{F}_{e}\left(\xi, t^{\prime}\right) d t^{\prime} d \xi \\
& =-i \int_{t^{\prime}=0}^{t} S\left[F_{e}\left(\cdot, t^{\prime}\right) ; 0\right]\left(x, t-t^{\prime}\right) d t^{\prime}
\end{aligned}
$$

where $\widehat{F}_{e}$ is the Fourier transform of $F_{e}$ with respect to $x$, and $S\left[F_{e}\left(\cdot, t^{\prime}\right) ; 0\right]$ in the Duhamel representation (4.8) denotes the solution (4.3) of problem $\mathrm{A}_{1} \operatorname{IVP}(4.2)$ with $F_{e}\left(x, t^{\prime}\right)$ in place of the initial datum $U_{0}(x)$.

\section{$B_{2}$. The homogeneous linear IBVP with zero initial condition}

$$
\begin{array}{ll}
v_{t}+v_{x x x}=0, & x \in(0, \infty), t \in(0, T), \\
v(x, 0)=0, & x \in[0, \infty), \\
v(0, t)=-W(0, t) \doteq-W_{0}(t), & t \in[0, T],
\end{array}
$$

whose solution is $v(x, t) \doteq S\left[0,-W_{0} ; 0\right](x, t)$, which is defined in $(2.3)$.

Next, we describe the estimates for each one of the above problems.

Theorem 4.1 (Estimates for homogeneous IVP $\mathrm{A}_{1}$ ). The solution $U=S\left[U_{0} ; 0\right]$ to IVP (4.2) defined by formula (4.3) satisfies the space estimate

$$
\sup _{t \in[0, T]}\left\|S\left[U_{0} ; 0\right](t)\right\|_{H_{x}^{s}(\mathbb{R})}=\left\|U_{0}\right\|_{H_{x}^{s}(\mathbb{R})}, \quad s \in \mathbb{R}
$$

the time estimate

$$
\sup _{x \in \mathbb{R}}\left\|S\left[U_{0} ; 0\right](x)\right\|_{H_{t}^{\frac{s+1}{3}}(0, T)} \leqslant c_{s}(1+\sqrt{T})\left\|U_{0}\right\|_{H_{x}^{s}(\mathbb{R})}, \quad s \geqslant-1,
$$

and the time estimate for the derivative of the solution

$$
\sup _{x \in \mathbb{R}}\left\|\partial_{x} S\left[U_{0} ; 0\right](x)\right\|_{H_{t}^{\frac{s}{3}}(0, T)} \leqslant c_{s}(1+\sqrt{T})\left\|U_{0}\right\|_{H_{x}^{s}(\mathbb{R})}, \quad s \geqslant 0 .
$$

The proof of this result can be found in Ref. 20 except the derivative time estimate (4.12). However, its proof is similar to that of the time estimate (4.11), and therefore, we omit it.

The solution to the IBVP $A_{2}$ is estimated by first extending $G_{0}$ from $(0, T)$ to a function $h$ on $\mathbb{R}$ supported in $[0,2]$ and such that $\|h\|_{H_{t}^{\frac{s+1}{3}(\mathbb{R})}} \lesssim\left\|G_{0}\right\|_{H_{t}^{\frac{s+1}{3}}(0, T)}$, via Lemma 2.1, and then estimating the solution $v=S[0, h ; 0]$ to the following pure IBVP:

$$
\begin{array}{ll}
u_{t}+u_{x x x}=0, & x \in(0, \infty), t \in(0,2), \\
u(x, 0)=0, & x \in[0, \infty), \\
u(0, t)=h(t), & t \in[0,2] .
\end{array}
$$
by

Theorem 4.2 (Estimates for pure IBVP on the half-line). Suppose that $\frac{1}{2}<s<\frac{3}{2}$. The solution $v=S[0, h ; 0]$ to pure IBVP (4.13) defined

$$
v(x, t)=S[0, h ; 0] \doteq-\frac{1}{2 \pi} \int_{\partial D^{+}} e^{i k x+i k^{3} t} 3 k^{2} \hat{h}\left(k^{3}\right) d k, \quad x \in[0, \infty), t \in[0,2]
$$


satisfies the space estimate

$$
\sup _{t \in[0,2]}\|S[0, h ; 0](t)\|_{H_{x}^{s}(0, \infty)} \leqslant c_{s}\|h\|_{H_{t}^{\frac{s+1}{3}}(\mathbb{R})}
$$

the time estimate

$$
\sup _{x \in[0, \infty)}\|S[0, h ; 0](x)\|_{H_{t}^{\frac{s+1}{3}}(0,2)} \leqslant c_{s}(1+\sqrt{T})\|h\|_{H_{t}^{\frac{s+1}{3}}(\mathbb{R})}
$$

and the time estimate for the derivative of the solution

$$
\sup _{x \in[0, \infty)}\left\|\partial_{x} S[0, h ; 0](x)\right\|_{H_{t}^{\frac{5}{3}}(0,2)} \leqslant c_{s}(1+\sqrt{T})\|h\|_{H_{t}^{\frac{s+1}{3}}(\mathbb{R})} .
$$
omit it.

Also, the proof of this result can be found in Ref. 20 except estimate (4.17), which can be proved like estimate (4.16), and therefore, we

Theorem 4.3 (Estimates for forced IVP B1). The solution $W=S\left[0 ; F_{e}\right]$ of the forced IVP (4.6) defined by Eqs. (4.7) and (4.8) satisfies the space estimate

$$
\sup _{t \in[0, T]}\left\|S\left[0 ; F_{e}\right](t)\right\|_{H_{x}^{s}(\mathbb{R})} \leqslant \int_{t=0}^{T}\left\|F_{e}(t)\right\|_{H_{x}^{s}(\mathbb{R})} d t, \quad s \in \mathbb{R}
$$

the time estimate

$$
\sup _{x \in \mathbb{R}}\left\|S\left[0 ; F_{e}\right](x)\right\|_{H_{t}^{\frac{s+1}{3}}(0, T)} \lesssim \begin{cases}\max \left\{T^{\frac{1}{2}}, T^{\frac{1-2 s}{3}}\right\}\left\|F_{e}\right\|_{L^{2}\left([0, T] ; H_{x}^{s}(\mathbb{R})\right),} & -1 \leqslant s<\frac{1}{2} \\ T^{\frac{2-s}{3}}\left\|F_{e}\right\|_{L^{2}\left([0, T] ; H_{x}^{s}(\mathbb{R})\right)}, & \frac{1}{2}<s<2\end{cases}
$$

and the time estimate for the derivative of solution

$$
\sup _{x \in \mathbb{R}}\left\|\partial_{x} S\left[0 ; F_{e}\right](x)\right\|_{H_{t}^{\frac{s}{3}}(0, T)} \lesssim\left\{\begin{array}{ll}
\max \left\{T^{\frac{1}{2}}, T^{\frac{3-2 s}{3}}\right\}\left\|F_{e}\right\|_{L^{2}\left([0, T] ; H_{x}^{s}(\mathbb{R})\right)}, & 0 \leqslant s<\frac{3}{2} \\
T^{\frac{3-s}{3}}\left\|F_{e}\right\|_{L^{2}\left([0, T] ; H_{x}^{s}(\mathbb{R})\right)}, & \frac{3}{2}<s<3
\end{array} .\right.
$$

Estimates for problem $\mathbf{B}_{2}$. By the time estimate (4.19), we have that $-W_{0}(t) \in H_{t}^{\frac{s+1}{3}}(0, T)$, and similarly by the time estimate (4.11), we have that $G_{0}(t) \in H_{t}^{\frac{s+1}{3}}(0, T)$. Therefore, the solution of problem $B_{2}$ (like that of problem $A_{2}$ ) is estimated by using Theorem 4.2.

Next, combining the above estimates, we prove Theorem 2.1.

Proof of Theorem 2.1. Since the space estimate is done in Ref. 20, here, we focus on the time estimate of the solution. The time estimate for the derivative of the solution is similar. Using the superposition principle, we express the Fokas solution formula for the half-line forced IBVP (2.1) as follows when $x>0$ and $0<t<T$ :

$$
S\left[w_{0}, g_{0} ; F\right]=S\left[U_{0} ; 0\right]+S\left[0, g_{0}-\left.S\left[U_{0} ; 0\right]\right|_{x=0} ; 0\right]+S\left[0 ; F_{e}\right]+S\left[0,-\left.S\left[0 ; F_{e}\right]\right|_{x=0} ; 0\right] .
$$

Let $m=\frac{s+1}{3}$. Applying the triangular inequality for the norm $\sup _{x \geqslant 0}\|\cdot\|_{H_{t}^{m}(0, T)}$, then using the fact that $T<1$ and $(0, \infty) \subset \mathbb{R}$, and finally applying the time estimate (4.11), (4.16), and (4.19), from (4.21), we get

$$
\begin{aligned}
\sup _{x \geqslant 0}\left\|S\left[w_{0}, g_{0} ; F\right](x)\right\|_{H_{t}^{m}(0, T)} & \leqslant \sup _{x \in \mathbb{R}}\left\|S\left[U_{0} ; 0\right](x)\right\|_{H_{t}^{m}(0, T)}+\sup _{x \geqslant 0}\left\|S\left[0, g_{0}-\left.S\left[U_{0} ; 0\right]\right|_{x=0} ; 0\right](x)\right\|_{H_{t}^{m}(0,2)} \\
& +\sup _{x \in \mathbb{R}}\left\|S\left[0 ; F_{e}\right](x)\right\|_{H_{t}^{m}(0, T)}+\sup _{x \geqslant 0}\left\|S\left[0,-\left.S\left[0 ; F_{e}\right]\right|_{x=0} ; 0\right](x)\right\|_{H_{t}^{m}(0,2)} \\
& \leqslant c_{s}\left(\left\|U_{0}\right\|_{H_{x}^{s}(\mathbb{R})}+\left\|g_{0}\right\|_{H_{t}^{\frac{s+1}{3}}(0, T)}+T^{\frac{2-s}{3}}\left\|F_{e}\right\|_{L^{2}\left([0, T] ; H_{x}^{s}(\mathbb{R})\right)}\right) .
\end{aligned}
$$

Now, using the estimates $\left\|U_{0}\right\|_{\left.H_{x}^{s}(\mathbb{R})\right)} \lesssim\left\|w_{0}\right\|_{H_{x}^{s}(0, \infty)}$ and $\left\|F_{e}\right\|_{\left.H_{x}^{s}(\mathbb{R})\right)} \lesssim\|F\|_{H_{x}^{s}(0, \infty)}$ gives the desired time estimate (2.5). 
Proof of Theorem 4.3. Here, we only prove the time estimate (4.19). The time estimate (4.20) is similar, and the proof of the space estimate can be found in Ref. 20. Noting that $-1 \leq s<2, s \neq \frac{1}{2} \Longleftrightarrow 0 \leq m<1, m \neq \frac{1}{2}$, we use the following physical space description of $H_{t}^{m}(0, T)$ norm [see (3.9)]:

$$
\|W(x)\|_{H_{t}^{m}(0, T)}^{2}=\|W(x)\| W(x) L_{t}^{2}(0, T)^{2}+2 \int_{t=0}^{T} \int_{z=0}^{T-t} \frac{|W(x, z+t)-W(x, t)|^{2}}{z^{1+2 \beta}} d z d t .
$$

Next, we shall estimate the $L^{2}$-norm $\|W(x)\|_{L_{t}^{2}(0, T)}$ and the fractional norm $\|W(x)\|_{\beta}$.

Estimation of the $L^{2}$-norm $\|W(x)\|_{L_{t}^{2}(0, T)}$. Using the Duhamel representation (4.8) of the solution to the forced linear IVP with zero initial conditions (4.6), we have

$$
\|W(x)\|_{L_{t}^{2}(0, T)}=\left(\int_{t=0}^{T}\left(\int_{t^{\prime}=0}^{t}\left|S\left[F_{e}\left(\cdot, t^{\prime}\right) ; 0\right]\left(x, t-t^{\prime}\right)\right| d t^{\prime}\right)^{2} d t\right)^{\frac{1}{2}} .
$$

In the inside integral, replacing the upper limit $t$ with $T$ and applying Minkowski's inequality to switch $d t$ and $d t^{\prime}$, we get

$$
\|W(x)\|_{L_{t}^{2}(0, T)} \leqslant \int_{t^{\prime}=0}^{T}\left\|S\left[F_{e}\left(\cdot, t^{\prime}\right) ; 0\right]\left(x, t-t^{\prime}\right)\right\|_{L_{t}^{2}(0, T)} d t^{\prime}
$$

Now, using the time estimate for the homogeneous IVP (4.11) with $m=0$ or $s=-1$, we obtain

$$
\sup _{x \in \mathbb{R}}\|W(x)\|_{L_{t}^{2}(0, T)} \lesssim \int_{t=0}^{T}\left\|F_{e}(t)\right\|_{H_{x}^{s}(\mathbb{R})} d t \leqslant \sqrt{T}\left(\int_{t=0}^{T}\left\|F_{e}(t)\right\|_{H_{x}^{s}(\mathbb{R})}^{2} d t\right)^{\frac{1}{2}}, s \geqslant-1,
$$

where in the last step we applied the Cauchy-Schwarz inequality.

Estimation of the fractional norm $\|W(x)\|_{\beta}, 0<\beta<1$. Using solution formula (4.8), we have

$$
\begin{aligned}
W(x, t+z)-W(x, t) & \simeq \int_{t^{\prime}=0}^{t}\left[S\left[F_{e}\left(\cdot, t^{\prime}\right) ; 0\right]\left(x, t+z-t^{\prime}\right)-S\left[F_{e}\left(\cdot, t^{\prime}\right) ; 0\right]\left(x, t-t^{\prime}\right)\right] d t^{\prime} \\
& +\int_{t^{\prime}=t}^{t+z} S\left[F_{e}\left(\cdot, t^{\prime}\right) ; 0\right]\left(x, t+z-t^{\prime}\right) d t^{\prime} .
\end{aligned}
$$

Now, using the inequality $(a+b)^{2} \leqslant 2 a^{2}+2 b^{2}$, the fractional part in (4.22) yields

$$
\begin{aligned}
\|W(x)\|_{\beta}^{2} & \lesssim \int_{t=0}^{T} \int_{z=0}^{T-t} \frac{\left|\int_{t^{\prime}=0}^{t}\left[S\left[F_{e}\left(\cdot, t^{\prime}\right) ; 0\right]\left(x, t+z-t^{\prime}\right)-S\left[F_{e}\left(\cdot, t^{\prime}\right) ; 0\right]\left(x, t-t^{\prime}\right)\right] d t^{\prime}\right|^{2}}{z^{1+2 \beta}} d z d t \\
& +\int_{t=0}^{T} \int_{z=0}^{T-t} \frac{1}{z^{1+2 \beta}}\left|\int_{t^{\prime}=t}^{t+z} S\left[F_{e}\left(\cdot, t^{\prime}\right) ; 0\right]\left(x, t+z-t^{\prime}\right) d t^{\prime}\right|^{2} d z d t .
\end{aligned}
$$

Next, we estimate (4.25a) and (4.25b) separately. We begin by estimating (4.25a). We have

$$
(4.25 a) \leqslant \int_{t=0}^{T} \int_{z=0}^{T-t}\left(\frac{1}{z^{\frac{1+2 \beta}{2}}} \int_{t^{\prime}=0}^{T}\left|S\left[F_{e}\left(\cdot, t^{\prime}\right) ; 0\right]\left(x, t+z-t^{\prime}\right)-S\left[F_{e}\left(\cdot, t^{\prime}\right) ; 0\right]\left(x, t-t^{\prime}\right)\right| d t^{\prime}\right)^{2} d z d t .
$$

Applying Minkowski's inequality to switch $d z d t$ and $d t^{\prime}$, we get

$$
(4.25 \mathrm{a}) \leqslant\left(\int_{t^{\prime}=0}^{T}\left(\int_{t=0}^{T} \int_{z=0}^{T-t} \frac{1}{z^{1+2 \beta}}\left|S\left[F_{e}\left(\cdot, t^{\prime}\right) ; 0\right]\left(x, t+z-t^{\prime}\right)-S\left[F_{e}\left(\cdot, t^{\prime}\right) ; 0\right]\left(x, t-t^{\prime}\right)\right|^{2} d z d t\right)^{\frac{1}{2}} d t^{\prime}\right)^{2},
$$

which by the fractional norm description (3.10) reads as follows:

$$
(4.25 \mathrm{a}) \simeq\left(\int_{t^{\prime}=0}^{T}\left\|S\left[F_{e}\left(\cdot, t^{\prime}\right) ; 0\right]\left(x, t-t^{\prime}\right)\right\|_{\beta} d t^{\prime}\right)^{2} .
$$


Finally, applying the time estimate for the homogeneous IVP (4.11) with $\frac{s+1}{3}=\beta$, we get

$$
(4.25 \mathrm{a}) \lesssim\left(\int_{t^{\prime}=0}^{T}\left\|F_{e}\left(t^{\prime}\right)\right\|_{H_{x}^{s}(\mathbb{R})} d t^{\prime}\right)^{2} \leqslant T \int_{t=0}^{T}\left\|F_{e}(t)\right\|_{H_{x}^{s}(\mathbb{R})}^{2} d t,
$$

which is the desired bound for (4.25a).

Next, we estimate (4.25b) for which we consider the cases $0<\beta<\frac{1}{2}$ and $\frac{1}{2}<\beta<1$ separately.

The case $\frac{1}{2}<\beta<1$ or $\frac{1}{2}<s<2$. As we will see below, in this case, we utilize the Sobolev embedding theorem. Using solution formula (4.3) to the initial value problem with data $F_{e}(x, t)$, and switching the $d \xi$ and $d t^{\prime}$ integrations, we get

$$
(4.25 \mathrm{~b})=\int_{t=0}^{T} \int_{z=0}^{T-t} \frac{1}{z^{1+2 \beta}}\left|\frac{1}{2 \pi} \int_{\xi \in \mathbb{R}} e^{i \xi x+i \xi^{3}(t+z)} \int_{t^{\prime}=t}^{t+z} e^{-i \xi^{3} t^{\prime}} \widehat{F}_{e}\left(\xi, t^{\prime}\right) d t^{\prime} d \xi\right|^{2} d z d t .
$$

Also, using the fact that for any non-negative function $\phi(x, t)$ we have $\int_{t} \phi(x, t) d t \leqslant \int_{t}\|\phi(t)\|_{L_{x}^{\infty}} d t$, the last relation gives

$$
(4.25 b) \leqslant \int_{t=0}^{T} \int_{z=0}^{T-t} \frac{1}{z^{1+2 \beta}}\left\|\frac{1}{2 \pi} \int_{\xi \in \mathbb{R}} e^{i \xi x+i \xi^{3}(t+z)} \int_{t^{\prime}=t}^{t+z} e^{-i \xi^{3} t^{\prime}} \widehat{F}_{e}\left(\xi, t^{\prime}\right) d t^{\prime} d \xi\right\|_{L_{x}^{\infty}(\mathbb{R})}^{2} d z d t .
$$

Furthermore, applying the Sobolev embedding theorem, we bound the $L^{\infty}$-norm by the $H^{s}$-norm as follows, when $s>\frac{1}{2}$ :

$$
(4.25 b) \leqslant \int_{t=0}^{T} \int_{z=0}^{T-t} \frac{1}{z^{1+2 \beta}}\left\|\frac{1}{2 \pi} \int_{\xi \in \mathbb{R}} e^{i \xi x} e^{i \xi^{3}(t+z)} \int_{t^{\prime}=t}^{t+z} e^{-i \xi^{3} t^{\prime}} \widehat{F}_{e}\left(\xi, t^{\prime}\right) d t^{\prime} d \xi\right\|_{H_{x}^{s}(\mathbb{R})}^{2} d z d t .
$$

Then, computing the $H^{s}$-norm, we get

$$
(4.25 \mathrm{~b}) \leqslant \int_{t=0}^{T} \int_{z=0}^{T-t} \frac{1}{z^{1+2 \beta}} \int_{\xi \in \mathbb{R}}\left(1+\xi^{2}\right)^{s}\left|\int_{t^{\prime}=t}^{t+z} e^{-i \xi^{3} t^{\prime}} \widehat{F}_{e}\left(\xi, t^{\prime}\right) d t^{\prime}\right|^{2} d \xi d z d t,
$$

which by using Minkowski's inequality to interchange $d \xi$ and $d t^{\prime}$ gives

$$
(4.25 \mathrm{~b}) \leqslant \int_{t=0}^{T} \int_{z=0}^{T-t} \frac{1}{z^{1+2 \beta}}\left(\int_{t^{\prime}=t}^{t+z}\left\|F_{e}\left(t^{\prime}\right)\right\|_{H_{x}^{s}(\mathbb{R})} d t^{\prime}\right)^{2} d z d t .
$$

Moreover, applying the Cauchy-Schwarz inequality in $t^{\prime}$, we get

$$
(4.25 b) \leqslant \int_{t=0}^{T} \int_{z=0}^{T-t} \int_{t^{\prime}=t}^{t+z}\left\|F_{e}\left(t^{\prime}\right)\right\|_{H_{x}^{s}(\mathbb{R})}^{2} z^{-2 \beta} d t^{\prime} d z d t
$$

and using Fubini's theorem to interchange $d t^{\prime}$ and $d t$ integrations, we have

$$
(4.25 \mathrm{~b}) \leqslant \int_{t^{\prime}=0}^{T}\left\|F_{e}\left(t^{\prime}\right)\right\|_{H_{x}^{s}(\mathbb{R})}^{2} \int_{z=0}^{T} z^{-2 \beta} \int_{t=t^{\prime}-z}^{t^{\prime}} d t d z d t^{\prime} \stackrel{\beta<1}{=} \frac{T^{2-2 \beta}}{2-2 \beta} \int_{t^{\prime}=0}^{T}\left\|F_{e}\left(t^{\prime}\right)\right\|_{H_{x}^{s}(\mathbb{R})}^{2} d t^{\prime},
$$

which is the desired time estimate for the second part of fractional norm (4.25b) when $\frac{1}{2}<s<2$. Finally, combining the $L^{2}$ estimate (4.23) with the fractional case estimates (4.27) and (4.31), and recalling that $1-\beta=\frac{2-s}{3}$, we obtain

$$
\begin{aligned}
\sup _{x \in \mathbb{R}}\|W(x)\|_{H_{t}^{m}(0, T)} & \lesssim \sqrt{T}\left(\int_{t=0}^{T}\left\|F_{e}(t)\right\|_{H_{x}^{s}(\mathbb{R})}^{2} d t\right)^{\frac{1}{2}}+T^{1-\beta}\left(\int_{t=0}^{T}\left\|F_{e}(t)\right\|_{H_{x}^{s}(\mathbb{R})}^{2} d t\right)^{\frac{1}{2}} \\
& \stackrel{T<1}{\lesssim} T^{\frac{2-s}{3}}\left(\int_{t=0}^{T}\left\|F_{e}(t)\right\|_{H_{x}^{s}(\mathbb{R})}^{2} d t\right)^{\frac{1}{2}},
\end{aligned}
$$

which is the desired time estimate (4.19) when $\frac{1}{2}<s<2$.

The case $0<\beta<\frac{1}{2}$ or $-1<s<\frac{1}{2}$. Applying the Cauchy-Schwarz inequality in the $d t^{\prime}$ integral of (4.25b), we get 


$$
(4.25 \mathrm{~b}) \leqslant \int_{t=0}^{T} \int_{z=0}^{T-t} \frac{1}{z^{1+2 \beta}}\left(z \int_{t^{\prime}=t}^{t+z}\left|S\left[F_{e}\left(\cdot, t^{\prime}\right) ; 0\right]\left(x, t+z-t^{\prime}\right)\right|^{2} d t^{\prime}\right) d z d t
$$

Now, simplifying, using Fubini's theorem to interchange $d z$ and $d t$ integrations, letting $t_{1}=t+z$, replacing lower limit $t_{1}=z$ with $t_{1}=0$, and again using Fubini's theorem to interchange $d t_{1}$ and $d t^{\prime}$ integrations, we get

$$
(4.25 b) \leqslant \frac{T^{1-2 \beta}}{1-2 \beta} \int_{t^{\prime}=0}^{T}\left\|S\left[F_{e}\left(\cdot, t^{\prime}\right) ; 0\right]\left(x, t_{1}-t^{\prime}\right)\right\|_{L_{t_{1}}^{2}\left(t^{\prime}, T\right)}^{2} d t^{\prime} .
$$

Furthermore, using the homogeneous IVP time estimate (4.11) with $s=-1$, we get

$$
(4.25 b) \leqslant \frac{1}{1-2 \beta} T^{1-2 \beta}\left\|F_{e}\right\|_{L^{2}\left([0, T] ; H_{x}^{s}(\mathbb{R})\right)}^{2}, \forall s \geqslant-1,
$$

which is the desired time estimate for the second part of fractional norm (4.25b) when $-1 \leqslant s<\frac{1}{2}$. obtain

Finally, combining the $L^{2}$-norm estimate (4.23) with the fractional case estimates (4.27) and (4.35), and recalling that $1-2 \beta=\frac{1-2 s}{3}$, we

$$
\begin{aligned}
\sup _{x \in \mathbb{R}}\|W(x)\|_{H_{t}^{m}(0, T)} & \lesssim \sqrt{T}\left(\int_{t=0}^{T}\left\|F_{e}(t)\right\|_{H_{x}^{s}(\mathbb{R})}^{2} d t\right)^{\frac{1}{2}}+T^{1-2 \beta}\left(\int_{t=0}^{T}\left\|F_{e}(t)\right\|_{H_{x}^{s}(\mathbb{R})}^{2} d t\right)^{\frac{1}{2}} \\
& \stackrel{T<1}{\lesssim} \max \left\{T^{\frac{1}{2}}, T^{\frac{1-2 s}{3}}\right\}\left(\int_{t=0}^{T}\left\|F_{e}(t)\right\|_{H_{x}^{s}(\mathbb{R})}^{2} d t\right)^{\frac{1}{2}}, \quad-1 \leqslant s<\frac{1}{2},
\end{aligned}
$$

which is the desired time estimate (4.19) when $-1 \leqslant s<\frac{1}{2}$.

\section{ESTIMATES IN THE SOLUTION SPACE $X$}

We begin by recalling that our iteration (solution) map defined by the forced linear IBVP with initial data $u_{0}$; boundary data $g_{0}, h_{0}, h_{1}$; and forcing $f$ reads as follows:

$$
u \mapsto \Phi u \doteq S\left[u_{0}, g_{0}, h_{0}, h_{1} ; f\right]=\left.S\left[w_{0}, g_{0} ; F\right]\right|_{x \in(0, \ell)}+S\left[0,0, h_{2}, h_{3} ; 0\right]
$$

Our strategy for proving existence of solution to our IBVP for $\mathrm{KdV}$ on the interval $(0, \ell)$ is to use the basic estimate $(1.23)$ with $f=u u_{x}$ and show that the iteration map $\Phi$ is a contraction in a good solution space. However, the term $\left(\int_{0}^{T}\|f\|_{H_{x}^{s}}^{2}(0, \ell) d t\right)^{\frac{1}{2}}$ is not good if we consider the classical Hadamard space $C\left([0, T] ; H_{x}^{s}(0, \ell)\right)$ as our solution space since replacing $f$ with the $\operatorname{KdV}$ nonlinearity $u w_{x}$ we would need the following "algebra property:"

$$
\|f\|_{H_{x}^{s}(0, \ell)}=\left\|u w_{x}\right\|_{H_{x}^{s}(0, \ell)} \leqslant\|u\|_{H_{x}^{s}(0, \ell)}\|w\|_{H_{x}^{s}(0, \ell)},
$$

which is not true, and therefore, the last term of estimate (1.23) does not allow us to close the loop in Hadamard space $C\left([0, T] ; H_{x}^{s}(0, \ell)\right)$. In the framework of the KdV IVP, this was first realized by Kenig, Ponce, and Vega in Ref. 33, where they introduced certain $\lambda$-norms in order to prove well-posedness of $\mathrm{KdV}$ for data in $H^{s}(\mathbb{R}), s>3 / 4$. These $\lambda$-norms were also used in Ref. 20 for proving well-posedness of the KdV on the half-line. The $\lambda$-norms needed for the interval are similar and are defined in the introduction by (1.8)-(1.10).

Next, we shall provide a bilinear estimate of the problem term by using the $\lambda$-norms. More precisely, we will prove the following result.

Lemma 5.1 (Bilinear estimates on an interval). Let $\frac{1}{2}<s<1$ and $0<T<1$. Then, for two test functions $u(x, t)$ and $w(x, t)$ defined for $0<x<\ell$ and $0<t<T$, we have

$$
\int_{0}^{T}\left\|u w_{x}(t)\right\|_{H_{x}^{s}(0, \ell)}^{2} d t \leqslant\left(\ell \cdot T^{\frac{1}{2}}+4 T^{\frac{1}{2}}\right) \lambda_{1, s}^{T}(u)^{2} \lambda_{3}^{T}(w)^{2}+4 \ell \cdot \lambda_{1, s}^{T}(u)^{2} \lambda_{2, s}^{T}(w)^{2} .
$$

Furthermore, in the space $X$, this bilinear estimate reads as follows:

$$
\left\|u w_{x}\right\|_{L^{2}\left([0, T] ; H_{x}^{s}(0, \ell)\right)} \doteq\left(\int_{0}^{T}\left\|u w_{x}\right\|_{H_{x}^{s}(0, \ell)}^{2} d t\right)^{\frac{1}{2}} \leqslant \max \left\{\left(\ell \cdot T^{\frac{1}{2}}+4 T^{\frac{1}{2}}\right)^{\frac{1}{2}}, 2 \ell^{\frac{1}{2}}\right\}\|u\|_{X}\|w\|_{X} .
$$


Proof of Lemma 5.1. The first bilinear estimate (5.2) implies the second bilinear estimate (5.3). This follows from the definition of the norm $\|\cdot\|_{X}$ in $(1.7)$, that is,

$$
\|u\|_{X} \doteq \lambda^{T}(u)=\max \left\{\lambda_{1, s}^{T}(u), \lambda_{2, s}^{T}(u), \lambda_{3}^{T}(u)\right\} .
$$

So, here, we focus only on deriving (5.2). By the fractional description of $H^{s}$-norm, we have

$$
\int_{0}^{T}\left\|u w_{x}(t)\right\|_{H_{x}^{s}(0, \ell)}^{2} d t=\int_{0}^{T}\left[\left\|u w_{x}(t)\right\|_{L_{x}^{2}(0, \ell)}^{2}+\left\|u w_{x}(t)\right\|_{\beta}^{2}\right] d t
$$

For the first term, taking sup over $x$ for both functions and then taking sup of $u(x, t)$ over $t$, we get

$$
\int_{0}^{T} \int_{0}^{\ell}\left|u(x, t) w_{x}(x, t)\right|^{2} d x d t \leqslant \ell \cdot \sup _{t \in[0, T]} \sup _{x \in[0, \ell]}|u(x, t)|^{2} \int_{0}^{T} \sup _{x \in[0, \ell]}\left|w_{x}(x, t)\right|^{2} d t .
$$

Then, by the Sobolev embedding theorem, for $s^{\prime}>\frac{1}{2}$, we have

$$
\sup _{x \in[0, \ell]}|u(x, t)| \leqslant\|u(x, t)\|_{H_{x}^{s^{\prime}}(0, \ell)} \leqslant \lambda_{1, s^{\prime}}^{T}(u)
$$

In addition, using the Cauchy-Schwarz inequality to $d t$, we have

$$
\int_{0}^{T} \sup _{x \in[0, \ell]}\left|w_{x}(x, t)\right|^{2} d t \leqslant\left(\int_{0}^{T} d t\right)^{\frac{1}{2}}\left(\int_{0}^{T} \sup _{x \in[0, \ell]}\left|w_{x}(x, t)\right|^{4} d t\right)^{\frac{1}{2}}=T^{\frac{1}{2}}\left[\lambda_{3}^{T}(w)\right]^{2} .
$$

Therefore, we get the desired estimate (5.2) for the first term of (5.4)

$$
\int_{0}^{T}\left\|u w_{x}(t)\right\|_{L_{x}^{2}(0, \ell)}^{2} d t \leqslant \ell \cdot T^{\frac{1}{2}}\left[\lambda_{1, s^{\prime}}^{T}(u) \lambda_{3}^{T}(w)\right]^{2}, \quad \forall s^{\prime}>\frac{1}{2} .
$$

For the second term in the right-hand side of (5.4), using the fractional norm definition, we have

$$
\begin{aligned}
\int_{0}^{T}\left\|u w_{x}(t)\right\|_{\beta}^{2} d t & \leqslant 2 \int_{0}^{T} \int_{0}^{\ell} \int_{0}^{\ell-x}\left|w_{x}(x+z, t)\right|^{2} \frac{|u(x+z, t)-u(x, t)|^{2}}{z^{1+2 s}} d z d x d t \\
& +2 \int_{0}^{T} \int_{0}^{\ell} \int_{0}^{\ell-x}|u(x, t)|^{2} \frac{\left|w_{x}(x+z, t)-w_{x}(x, t)\right|^{2}}{z^{1+2 s}} d z d x d t
\end{aligned}
$$

Furthermore, in the first $z$-integral letting $y=x+z$, we obtain

$$
\begin{aligned}
\int_{0}^{T}\left\|u w_{x}(t)\right\|_{\beta}^{2} d t & \leqslant 2 \int_{0}^{T} \sup _{y \in[0, \ell]}\left|w_{y}(y, t)\right|^{2} \int_{0}^{\ell} \int_{0}^{\ell} \frac{|u(x, t)-u(y, t)|^{2}}{|x-y|^{1+2 s}} d x d y d t \\
& +2 \int_{0}^{\ell} \sup _{t \in[0, T]}|u(x, t)|^{2} d x \cdot \sup _{x \in[0, \ell]} \int_{0}^{T} \int_{0}^{\ell-x} \frac{\left|w_{x}(x+z, t)-w_{x}(x, t)\right|^{2}}{z^{1+2 s}} d z d t .
\end{aligned}
$$

Using definitions (1.8) and (1.9), we get

$$
(5.9 \mathrm{~b}) \leqslant 2 \ell \cdot\left[\lambda_{1, s^{\prime}}^{T}(u) \lambda_{2, s}^{T}(w)\right]^{2}, \quad \forall s^{\prime}>\frac{1}{2} .
$$

Also, applying the Cauchy-Schwarz inequality in $t$, we get

$$
\begin{aligned}
(5.9 a) & \leqslant 2\left(\int_{0}^{T} \sup _{x \in[0, \ell]}\left|w_{x}(x, t)\right|^{4} d t\right)^{\frac{1}{2}} \cdot\left(\int_{0}^{T}\left(\int_{0}^{\ell} \int_{0}^{\ell} \frac{|u(x, t)-u(y, t)|^{2}}{|x-y|^{1+2 s}} d x d y\right)^{2} d t\right)^{\frac{1}{2}} \\
& =2 \lambda_{3}^{T}(w)^{2} \cdot\left(\int_{0}^{T}\|u\|_{H_{x}^{s}(0, \ell)}^{4} d t\right)^{\frac{1}{2}} \leqslant 2 T^{\frac{1}{2}}\left[\lambda_{3}^{T}(w) \lambda_{1, s}^{T}(u)\right]^{2} .
\end{aligned}
$$

Combining the inequalities above gives the desired estimate (5.2). 
Next, for proving that the solution map is a contraction on the space $X$, we need to estimate the iteration map $S\left[u_{0}, g_{0}, h_{0}, h_{1} ; u w_{x}\right]$ in the $\lambda$-norms (1.8)-(1.10).

Proposition 5.1 (Estimates for the forced linear IBVP in $X$ space). For $\frac{3}{4}<s<1$, the Fokas solution formula $S\left[u_{0}, g_{0}, h_{0}, h_{1} ; f\right]$ of the forced linear KdV IBVP defined by (1.18) with $f=u w_{x}$ satisfies the estimate

$$
\begin{aligned}
\left\|S\left[u_{0}, g_{0}, h_{0}, h_{1} ; u w_{x}\right]\right\|_{X} \leqslant c_{s}\left(\left\|u_{0}\right\|_{H_{x}^{s}(0, \ell)}+\left\|g_{0}\right\|_{H_{t}^{\frac{s+1}{3}}(0, T)}+\left\|h_{0}\right\|_{H_{t}^{\frac{s+1}{3}}(0, T)}+\left\|h_{1}\right\|_{H_{t}^{\frac{s}{3}}(0, T)}\right. \\
\left.+\max \left\{T^{\frac{1}{3}},\left(\ell \cdot T^{\frac{1}{2}}+4 T^{\frac{1}{2}}\right)^{\frac{1}{2}} \cdot T^{\frac{2-s}{3}}, 2 \ell^{\frac{1}{2}} \cdot T^{\frac{2-s}{3}}\right\}\|u\|_{X}\|w\|_{X}\right) .
\end{aligned}
$$

Proof of Proposition 5.1. Using the following decomposition of the solution to the linear forced IBVP (1.17) on the interval $(0, \ell)$

$$
S\left[u_{0}, g_{0}, h_{0}, h_{1} ; f\right]=\left.S\left[w_{0}, g_{0} ; F\right]\right|_{x \in(0, \ell)}+S\left[0,0, h_{2}, h_{3} ; 0\right],
$$

we see that it suffices to estimate the restriction of the solution of the half-line problem (2.1) and the solution to the pure linear IBVP on the interval (2.12).

Estimation of the $\lambda$-norm for $\left.S\left[w_{0}, g_{0} ; F\right]\right|_{x \in(0, \ell)}$. First, we recall the $\Lambda$-norms for the half-line defined in Ref. 20. They are given by

$$
\begin{aligned}
& \Lambda_{1, s}^{T}(w(t))=\sup _{t \in[0, T]}\|w(t)\|_{H_{x}^{s}(0, \infty)}, \\
& \Lambda_{2, s}^{T}(w)=\left(\sup _{x \in[0, \infty]} \int_{0}^{T}\left|\mathscr{D}_{x}^{s} \partial_{x} w(x, t)\right|^{2} d t\right)^{\frac{1}{2}}, \\
& \Lambda_{4}^{T}(w)=\left(\int_{0}^{T} \sup _{x \in[0, \infty]}\left|\partial_{x} w(x, t)\right|^{4} d t,\right)^{\frac{1}{4}},
\end{aligned}
$$

where the operator $\mathscr{D}_{x}^{s}$ is defined as

$$
\left|\mathscr{D}_{x}^{s} w(x, t)\right|^{2} \doteq \begin{cases}\left|\partial_{x}^{s} w(x, t)\right|^{2}, & s \in \mathbb{N} \\ \int_{0}^{\infty} \frac{\left|\partial_{x}^{\lfloor s\rfloor} w(x+z, t)-\partial_{x}^{\lfloor s\rfloor} w(x, t)\right|^{2}}{z^{1+2 \beta}} d z, & s=\lfloor s\rfloor+\beta, 0<\beta<1 .\end{cases}
$$

Also, we recall the following estimates proved in Ref. 20. For $\frac{1}{2}<s<1$, we have

$$
\Lambda_{1, s}^{T}\left(S\left[w_{0}, g_{0} ; F\right]\right) \leqslant c_{s}\left(\left\|w_{0}\right\|_{H_{x}^{s}(0, \infty)}+\left\|g_{0}\right\|_{H_{t}^{\frac{s+1}{3}}(0, T)}+T^{\frac{1}{3}}\|F\|_{L^{2}\left([0, T] ; H_{x}^{s}(0, \infty)\right)}\right)
$$

for $0 \leqslant s<1$, we have

$$
\Lambda_{2, s}^{T}\left(S\left[w_{0}, g_{0} ; F\right]\right) \lesssim\left\|w_{0}\right\|_{H_{x}^{s}(0, \infty)}+\left\|g_{0}\right\|_{H_{t}^{s+1}(0, T)}+T^{\frac{1}{3}}\|F\|_{L^{2}\left([0, T] ; H_{x}^{s}(0, \infty)\right)}
$$

and for $s \geqslant \frac{3}{4}$, we have

$$
\Lambda_{4}^{T}\left(S\left[w_{0}, g_{0} ; F\right]\right) \lesssim\left\|w_{0}\right\|_{H_{x}^{s}(0, \infty)}+\left\|g_{0}\right\|_{H_{t}^{\frac{s+1}{3}}(0, T)}+T^{\frac{1}{3}}\|F\|_{L^{2}\left([0, T] ; H_{x}^{s}(0, \infty)\right)}
$$

Using these estimates and the fact that $\left.S\left[w_{0}, g_{0} ; F\right]\right|_{x \in(0, \ell)}$ is a restriction of the function $S\left[w_{0}, g_{0} ; F\right]$ on the interval $[0, \ell]$, we obtain 


$$
\begin{aligned}
\lambda^{T}\left(\left.S\left[w_{0}, g_{0} ; F\right]\right|_{x \in(0, \ell)}\right) & \leqslant \lambda_{1, S}^{T}\left(\left.S\left[w_{0}, g_{0} ; F\right]\right|_{x \in(0, \ell)}\right)+\lambda_{2, s}^{T}\left(\left.S\left[w_{0}, g_{0} ; F\right]\right|_{x \in(0, \ell)}\right)+\lambda_{3}^{T}\left(\left.S\left[w_{0}, g_{0} ; F\right]\right|_{x \in(0, \ell)}\right) \\
& \leqslant \Lambda_{1, S}^{T}\left(S\left[w_{0}, g_{0} ; F\right]\right)+\Lambda_{2, s}^{T}\left(S\left[w_{0}, g_{0} ; F\right]\right)+\Lambda_{4}^{T}\left(S\left[w_{0}, g_{0} ; F\right]\right) \\
& \leqslant\left\|w_{0}\right\|_{H_{x}^{s}(0, \infty)}+\left\|g_{0}\right\|_{H_{t}^{\frac{s+1}{3}}(0, T)}+T^{\frac{1}{3}}\|F\|_{L^{2}\left([0, T] ; H_{x}^{s}(0, \infty)\right)} .
\end{aligned}
$$

Also, since $\left\|u_{0}\right\|_{H_{x}^{s}(0, \ell)}$ bounds $\left\|w_{0}\right\|_{H_{x}^{s}(0, \infty)}$ and $\|f(t)\|_{H_{x}^{s}(0, \ell)}$ bounds $\|F(t)\|_{H_{x}^{s}(0, \infty)}$, we get for $\frac{3}{4} \leqslant s<1$

$$
\lambda^{T}\left(\left.S\left[w_{0}, g_{0} ; F\right]\right|_{x \in(0, \ell)}\right) \lesssim\left\|u_{0}\right\|_{H_{x}^{s}(0, \ell)}+\left\|g_{0}\right\|_{H_{t}^{\frac{s+1}{3}(0, T)}}+T^{\frac{1}{3}}\|f\|_{L^{2}\left([0, T] ; H_{x}^{s}(0, \ell)\right)} .
$$

Finally, letting $f=u w_{x}$ and applying the bilinear estimate (5.3), we get the desired estimate (5.12) for the first term of (5.13).

Estimation of the $\lambda$-norm for $S\left[0,0, h_{2}, h_{3} ; 0\right]$. We do it for each one of the norms $\lambda_{1, s}^{T}, \lambda_{2, s}^{T}, \lambda_{3}^{T}$.

$\lambda_{1, s}^{T}$ - norm. By the definition of the $\lambda_{1, s}^{T}$-norm (1.8) and Theorem (1.2) (or Theorem 2.2), we have

$$
\lambda_{1, s}^{T}\left(S\left[0,0, h_{2}, h_{3} ; 0\right]\right) \leqslant c_{s}\left(\left\|h_{2}\right\|_{H_{t}^{\frac{s+1}{3}}(0,2)}+\left\|h_{3}\right\|_{H_{t}^{\frac{s}{3}}(0,2)}\right), \quad \frac{1}{2}<s<\frac{3}{2},
$$

which is bounded by the right-hand side of the estimate (5.12), as desired.

$\lambda_{2, s}^{T}-$ norm. Next, we shall prove that for $0 \leqslant s<1$, we have the desired estimate, i.e.,

$$
\lambda_{2, s}^{T}\left(S\left[0,0, h_{2}, h_{3} ; 0\right]\right) \leqslant c_{s}\left(\left\|h_{2}\right\|_{H_{t}^{\frac{s+1}{3}}(0,2)}+\left\|h_{3}\right\|_{H_{t}^{\frac{s}{3}}(0,2)}\right) .
$$

As in the Proof of Theorem 2.2 for the reduced IBVP, we estimate the part of the solution $S\left[0,0, h_{2}, h_{3} ; 0\right]$ that corresponds to $\partial D^{+}$and the boundary value $h_{3}$, i.e., $v_{11}=V_{0}+V_{1}+V_{2}$, which is given in (3.7). In fact, we only estimate $V_{2}$ which is given in (3.8) and the part of $V_{0}$, which is given by (3.19) and is denoted by $V_{0,2}$. We begin with $V_{2}$.

$\lambda_{2, s}^{T}\left(V_{2}\right)$. The case $\boldsymbol{s}=\mathbf{0}$. Using the formula for $V_{2}$, the definition of the $\lambda_{2}$-norm and making the following change of variables $\eta \doteq-k^{3}$, we get

$$
\lambda_{2,0}^{T}\left(V_{2}\right) \simeq\left(\sup _{x \in[0, \ell]} \int_{0}^{T}\left|\int_{-\infty}^{-1} C\left(-a \eta^{\frac{1}{3}}\right) e^{i \eta t} e^{\frac{\sqrt{3}}{2} \eta^{\frac{1}{3}} x} e^{-i \frac{1}{2} \eta^{\frac{1}{3}} x} \hat{h}_{3}(\eta) d \eta\right|^{2} d t\right)^{\frac{1}{2}} .
$$

Considering the inside integral as the inverse Fourier transform of a $t$-function on $\mathbb{R}$ after multiplying the integrand by the characteristic function $\chi_{(-\infty,-1)}(\eta)$, and applying Parseval's theorem, we get

$$
\lambda_{2,0}^{T}\left(V_{2}\right) \lesssim\left(\sup _{x \in[0, \ell]} \int_{-\infty}^{-1}\left|C\left(-a \eta^{\frac{1}{3}}\right)\right|^{2}\left|\hat{h}_{3}(\eta)\right|^{2} d \eta\right)^{\frac{1}{2}} \leqslant\left(\sup _{x \in[0, \ell]} \int_{\mathbb{R}}\left|\hat{h}_{3}(\eta)\right|^{2} d \eta\right)^{\frac{1}{2}}=\left\|h_{3}\right\|_{L_{t}^{2}(\mathbb{R})}
$$

since $C(a k)$ is bounded for $|k| \geqslant 1$. This is the desired estimate (5.23) for $s=0$.

The case $\mathbf{0}<\boldsymbol{s}<\mathbf{1}$. Making again the change of variables $\eta \doteq-k^{3}$, we see that the fractional part of the $\lambda_{2, s}^{T}$-norm defined by (1.11) reads as follows:

$$
\left[\lambda_{2, s}^{T}\left(V_{2}\right)\right]^{2} \simeq \sup _{x \in[0, \ell]} \int_{0}^{\ell-x} \frac{1}{z^{1+2 s}} \int_{0}^{T}\left|\int_{-\infty}^{-1} C\left(-a \eta^{\frac{1}{3}}\right)\left(e^{-i a \eta^{\frac{1}{3}}(x+z)}-e^{-i a \eta^{\frac{1}{3}} x}\right) e^{i \eta t} \hat{h}_{3}(\eta) d \eta\right|^{2} d t d z .
$$

Also, as before using Parseval's theorem in $\eta$-variable and using the fact that $C(a k)$ is bounded, we have the following estimate for the inside integral:

$$
\int_{0}^{T}\left|\int_{-\infty}^{-1} C\left(-a \eta^{\frac{1}{3}}\right)\left(e^{-i a \eta^{\frac{1}{3}}(x+z)}-e^{-i a \eta^{\frac{1}{3}} x}\right) e^{i \eta t} \hat{h}_{3}(\eta) d \eta\right|^{2} d t \lesssim \int_{-\infty}^{-1}\left|e^{-i a \eta^{\frac{1}{3}}(x+z)}-e^{-i a \eta^{\frac{1}{3}} x}\right|^{2}\left|\hat{h}_{3}(\eta)\right|^{2} d \eta .
$$

Furthermore, by Lemma 3.1, we have

$$
\left|e^{-i a \eta^{\frac{1}{3}}(x+z)}-e^{-i a \eta^{\frac{1}{3}} x}\right| \leqslant \sqrt{2}\left(1+\frac{1}{\sqrt{3}}\right)\left|e^{\frac{\sqrt{3}}{2} \eta^{\frac{1}{3}}(x+z)}-e^{\frac{\sqrt{3}}{2} \eta^{\frac{1}{3}} x}\right| .
$$

Finally, using the above two inequalities, we see that $\lambda_{2, s}^{T}\left(V_{2}\right)$ satisfies the estimate 


$$
\begin{aligned}
{\left[\lambda_{2, s}^{T}\left(V_{2}\right)\right]^{2} } & \lesssim \sup _{x \in[0, \ell]} \int_{-\infty}^{-1}\left|\hat{h}_{3}(\eta)\right|^{2}\left(\int_{0}^{\ell-x} \frac{1}{z^{1+2 s}} \cdot\left(e^{\frac{\sqrt{3}}{2} \eta^{\frac{1}{3}}(x+z)}-e^{\frac{\sqrt{3}}{2} \eta^{\frac{1}{3}}}\right)^{2} d z\right) d \eta \\
& \lesssim \sup _{x \in[0, \ell]} \int_{-\infty}^{-1}\left|\hat{h}_{3}(\eta)\right|^{2}|\eta|^{\frac{2}{3} s} d \eta \leqslant\left\|h_{3}\right\|_{H_{t}^{\frac{5}{3}}(\mathbb{R})},
\end{aligned}
$$

where in the second step we use again the inequality (3.15) after some simplification in the $d z$ integral. This is, again, the desired estimate.

$\lambda_{2, \mathbf{s}}^{\mathrm{T}}\left(\mathbf{V}_{\mathbf{0}, 2}\right)$. The case $\boldsymbol{s}=\mathbf{0}$. Recalling that $V_{0,2}(x, t)$ is given in (3.19), we see (like in the Proof of Theorem 2.2) that $\left|\hat{h}_{3}\left(-k^{3}\right)\right| \leqslant\left\|h_{3}\right\|_{L_{t}^{2}(0,2)}$, which gives the desired estimate

$$
\lambda_{2,0}^{T}\left(V_{2}\right) \lesssim\left\|h_{3}\right\|_{L_{t}^{2}(0,2)} .
$$

The case $\mathbf{0}<\boldsymbol{s}<\mathbf{1}$. Parametrizing the integral in (3.19), we have

$$
V_{0,2}(x, t) \doteq \int_{0+0 \cdot i}^{0.5+\frac{\sqrt{3}}{2} i} C(k) e^{i k x+i k^{3} t} k \hat{h}_{3}\left(k^{3}\right) d k=\int_{0}^{1} C(a k) e^{i a k x-i k^{3} t}(a k) \hat{h}_{3}\left(-k^{3}\right) d k,
$$

where $a=e^{\frac{\pi}{3} i}$. Since $C(a k) \cdot k$ is bounded for $|k| \leqslant 1$, using the Cauchy-Schwarz inequality, we get

$$
\left|\partial_{x} V_{0,2}(x+z)-\partial_{x} V_{0,2}(x)\right|^{2} \lesssim \int_{0}^{1}\left|e^{i a k(x+z)}-e^{i a k x}\right|^{2} d k \cdot \int_{0}^{1}\left|\hat{h}_{3}\left(-k^{3}\right)\right|^{2} d k
$$

Now, substituting the above estimate into the $\lambda_{2, s}$-norm (1.9), we get

$$
\lambda_{2, s}^{T}\left(V_{0,2}\right) \leqslant\left[\sup _{x \in[0, \ell]} T \cdot \int_{0}^{\ell-x} \frac{1}{z^{1+2 s}} \int_{0}^{1}\left|e^{i a k(x+z)}-e^{i a k x}\right|^{2} d k d z\right]^{\frac{1}{2}} \cdot\left[\int_{0}^{1}\left|\hat{h}_{3}\left(-k^{3}\right)\right|^{2} d k\right]^{\frac{1}{2}} .
$$

Also using the fact $\left|e^{\text {iakx }}\right| \lesssim 1$ for $x \in[0, \ell]$ and (3.15), we get

$$
\int_{0}^{\ell-x} \frac{1}{z^{1+2 s}} \int_{0}^{1}\left|e^{i a k(x+z)}-e^{i a k x}\right|^{2} d k d z=\int_{0}^{1} \int_{0}^{\ell-x} \frac{\left|e^{i a k(x+z)}-e^{i a k x}\right|^{2}}{z^{1+2 s}} d z d k \lesssim \int_{0}^{1} k^{2 s} d k \leqslant 1
$$

Combining the last two inequalities gives the desired estimate $\lambda_{2, s}^{T}\left(V_{0,2}\right) \leqslant\left\|h_{3}\right\|_{L^{2}(\mathbb{R})}$.

$\lambda_{3}^{T}$ - norm. Next, we prove that for $\frac{3}{4} \leqslant s<1$ we have

$$
\lambda_{3}^{T}\left(S\left[0,0, h_{2}, h_{3} ; 0\right]\right) \leqslant c_{s}\left(\left\|h_{2}\right\|_{H_{t}^{3}(0,2)}+\left\|h_{3}\right\|_{H_{t}^{\frac{s}{3}}(0,2)}\right) .
$$

As before, here, we estimate only $V_{0,2}$ and $V_{2}$ since the estimation of other terms of the solution $S\left[0,0, h_{2}, h_{3} ; 0\right]$ is similar.

Estimation of $\boldsymbol{\lambda}_{\mathbf{3}}^{\mathrm{T}}\left(\mathbf{V}_{\mathbf{0}, \mathbf{2}}\right)$. Differentiating $V_{0,2}(x, t)$ with respect to $x$ in $(5.24)$, we get

$$
\partial_{x} V_{0,2}(x, t)=\int_{0}^{1} C(a k) e^{i a k x-i k^{3} t} k^{2} \hat{h}_{3}\left(-k^{3}\right) d k
$$

Then, using the fact that $\left|C(a k) e^{i a k x-i k^{3} t} k^{2}\right| \lesssim 1$ for $k \in[0,1]$ and $x \in[0, \ell]$, we have

$$
\begin{aligned}
\lambda_{3}^{T}\left(V_{0,2}\right) & \doteq\left(\int_{0}^{T} \sup _{x \in[0, \ell]}\left|\partial_{x} V_{0,2}(x, t)\right|^{4} d t\right)^{\frac{1}{4}}=\left(\int_{0}^{T} \sup _{x \in[0, \ell]}\left|\int_{0}^{1} C(a k) e^{i a k x-i k^{3} t} k^{2} \hat{h}_{3}\left(-k^{3}\right) d k\right|^{4} d t\right)^{\frac{1}{4}} \\
& \lesssim\left(\int_{0}^{T} \sup _{x \in[0, \ell]}\left[\int_{0}^{1}\left|\hat{h}_{3}\left(-k^{3}\right)\right| d k\right]^{4} d t\right)^{\frac{1}{4}} \leqslant T^{\frac{1}{4}}\left\|\hat{h}_{3}\right\|_{L^{\infty}(0,1)} \leqslant\left\|h_{3}\right\|_{L_{t}^{2}(0,2)},
\end{aligned}
$$

where the last inequality follows from (3.20). Thus, we get the desired estimate for $V_{0,2}(x, t)$. 
Estimation of $\lambda_{3}^{\mathrm{T}}\left(\mathbf{V}_{\mathbf{2}}\right)$. Here, we are motivated by the ideas presented in Ref. 33 about the KdV Cauchy problem. We begin by recalling that

$$
V_{2}(x, t) \doteq \int_{1}^{\infty} C(a k) e^{i a k x-i k^{3} t}(a k) \hat{h}_{3}\left(-k^{3}\right) d k
$$

Differentiating it with respect to $x$ and letting $\tau=k^{3}$, we have for $x \in(0, \ell)$ and $t \in(0, T)$

$$
\partial_{x} V_{2}(x, t)=\int_{1}^{\infty} C(a k) e^{i a k x-i k^{3} t} i(a k)^{2} \hat{h}_{3}\left(-k^{3}\right) d k \simeq \int_{1}^{\infty} C\left(a \tau^{\frac{1}{3}}\right) e^{i a \tau^{\frac{1}{3}} x-i \tau t} \hat{h}_{3}(-\tau) d \tau .
$$

Therefore, by the definition of the $\lambda_{3}^{T}$-norm, we have

$$
\lambda_{3}^{T}\left(V_{2}\right) \doteq\left(\int_{0}^{T} \sup _{x \in[0, \ell]}\left|\partial_{x} V_{2}(x, t)\right|^{4} d t\right)^{\frac{1}{4}}=\left(\int_{0}^{T} \sup _{x \in[0, \ell]}\left|\int_{1}^{\infty} e^{i a \tau^{\frac{1}{3}} x-i \tau t} C\left(a \tau^{\frac{1}{3}}\right) \hat{h}_{3}(-\tau) d \tau\right|^{4} d t\right)^{\frac{1}{4}}
$$

Next, we define

$$
\widehat{R}(\tau) \doteq\left\{\begin{array}{l}
\tau^{\frac{1}{4}} C\left(a \tau^{\frac{1}{3}}\right) \widehat{h}(-\tau), \quad \tau \geqslant 1, \quad \text { and } \quad R(t) \doteq \frac{1}{2 \pi} \int_{\mathbb{R}} e^{i \tau \tau} \widehat{R}(\tau) d \tau .
\end{array}\right.
$$

Also, for a given number $r$, we define the operator

$$
\Delta^{r} R(x, t) \doteq \int_{1}^{\infty} e^{i a \tau^{1 / 3} x} e^{i \tau t} \tau^{r} \widehat{R}(\tau) d \tau .
$$

Using these definitions, we see that $\lambda_{3}^{T}\left(V_{2}\right)$ reads as follows:

$$
\lambda_{3}^{T}\left(V_{2}\right) \simeq\left(\int_{\mathbb{R}}\left\|\Delta^{-1 / 4} R(x,-t)\right\|_{L_{x}^{\infty}(0, \ell)}^{4} d t\right)^{\frac{1}{4}} \doteq\left\|\Delta^{-\frac{1}{4}} R(x,-t)\right\|_{L^{4}\left[\mathbb{R}_{t} ; L_{x}^{\infty}(0, \ell)\right]^{\prime}},
$$

and the desired estimate follows from the following result.

Lemma 5.2 (Strichartz-type estimate for the interval). For $R \in L_{t}^{2}(\mathbb{R})$, the operator $\Delta^{r} R$ defined by Eq. (5.31) is bounded from $L_{t}^{2}$ into $L^{q}\left[\mathbb{R}_{t} ; L_{x}^{p}(0, \ell)\right]$, that is, it satisfies the estimate

$$
\left\|\Delta^{r} R(x,-t)\right\|_{L^{q}\left[\mathbb{R}_{t} ; L_{x}^{p}(0, \ell)\right]}=\left\|\Delta^{r} R(x, t)\right\|_{L^{q}\left[\mathbb{R}_{t} ; L_{x}^{p}(0, \ell)\right]} \leqslant C_{T}\|R\|_{L_{t}^{2}(\mathbb{R})}
$$

for some constant $C_{T}>0$, where $r=-\frac{1}{4}, p=\infty$, and $q=4$.

In fact, since $\left|C\left(a \tau^{\frac{1}{3}}\right)\right|$ is bounded for $\tau \geqslant 1$, applying Lemma 5.2 for $R$ given by (5.30), we have

$$
\lambda_{3}^{T}\left(V_{2}\right) \lesssim\|R\|_{L_{t}^{2}(\mathbb{R})} \lesssim\left(\int_{1}^{\infty} \tau^{\frac{1}{2}}\left|\hat{h}_{3}(-\tau)\right|^{2} d \tau\right)^{\frac{1}{2}} \lesssim\left(\int_{\mathbb{R}}\left|1+\tau^{2}\right|^{\frac{1}{4}}\left|\hat{h}_{3}(\tau)\right|^{2} d \tau\right)^{\frac{1}{2}}=\left\|h_{3}\right\|_{H_{t}^{\frac{1}{4}}(\mathbb{R})} \leqslant\left\|h_{3}\right\|_{H_{t}^{\frac{5}{3}}(\mathbb{R})},
$$

if $s \geqslant \frac{3}{4}$. This proves the desired estimate (5.25).

Proof of Lemma 5.2. By duality, for $\frac{1}{p}+\frac{1}{p^{\prime}}=1$ and $\frac{1}{q}+\frac{1}{q^{\prime}}=1$, we have

$$
\left\|\Delta^{r} R(x, t)\right\|_{L^{q}\left[\mathbb{R}_{t} ; L_{x}^{p}(0, \ell)\right]}=\sup \left\{\left|\int\left[\Delta^{r} R(x, t)\right][Q(x, t)] d x d t\right|:\|Q\|_{L_{t}^{q^{\prime}}\left[\mathbb{R} ; L_{x}^{p^{\prime}}(0, \ell)\right]}=1\right\} .
$$

By Fubini's theorem, Plancherel's theorem, and Cauchy-Schwarz inequality, we see that

$$
\left|\int\left[\Delta^{r} R(x, t)\right][Q(x, t)] d x d t\right| \leqslant\|R\|_{L_{t}^{2}} \cdot\left\|\int_{x=0}^{\ell} \int_{\tau=1}^{\infty} e^{-i \tau t} e^{i a \tau^{1 / 3} x} \tau^{r} \widehat{Q}(x,-\tau) d \tau d x\right\|_{L_{t}^{2}(\mathbb{R})} .
$$

Therefore, to prove (5.33), it suffices to show that

$$
\left\|\int_{x=0}^{\ell} \Delta^{r} Q(x, \cdot) d x\right\|_{L_{t}^{2}(\mathbb{R})} \lesssim\|Q\|_{L_{x}^{p^{\prime}}(0, \ell) L_{t}^{q^{\prime}}(\mathbb{R})} .
$$

Again by Fubini's theorem, Plancherel's theorem, and Holder's inequality, we have 


$$
\left\|\int_{x=0}^{\ell} \Delta^{r} Q(x, \cdot) d x\right\|_{L_{t}^{2}(\mathbb{R})}^{2} \leqslant\|Q\|_{L_{t}^{q^{\prime}}\left[\mathbb{R}: L_{x}^{p^{\prime}}[0, \ell]\right]}\left\|\int_{\tau=1}^{\infty} \int_{x_{2}=0}^{\ell} e^{i \tau t} e^{i a \tau^{1 / 3} x_{2}-i \bar{a} \tau^{1 / 3} x_{1}} \tau^{2 r} \widehat{Q}^{t}\left(x_{2}, \tau\right) d x_{2} d \tau\right\|_{L_{t}^{q}\left[\mathbb{R}: L_{x_{1}}^{p}[0, \ell]\right]^{\prime}},
$$

which shows that it is suffices to prove that

$$
\left\|\int_{\tau=1}^{\infty} \int_{x_{2}=0}^{\ell} e^{i \tau t} e^{i a \tau^{1 / 3} x_{2}-i \bar{a} \tau^{1 / 3} x_{1}} \tau^{2 r} \widehat{Q}^{t}\left(x_{2}, \tau\right) d x_{2} d \tau\right\|_{L_{t}^{q}\left[\mathbb{R}: L_{x_{1}}^{p}[0, \ell]\right]} \lesssim\|Q\|_{L_{t}^{q^{\prime}}\left[\mathbb{R}: L_{x}^{p^{\prime}}[0, \ell]\right]} .
$$

Now, recalling that $a=\frac{1}{2}+i \frac{\sqrt{3}}{2}$ and using the Fourier transform property $\widehat{f * g}=\widehat{f} \cdot \widehat{g}$, we see that

$$
\begin{aligned}
& \int_{\tau=1}^{\infty} \int_{x_{2}=0}^{\ell} e^{i \tau t} e^{i a \tau^{1 / 3} x_{2}-i \bar{a} \tau^{1 / 3} x_{1}} \tau^{2 r} \widehat{Q}^{t}\left(x_{2}, \tau\right) d x_{2} d \tau \\
\simeq & \int_{x_{2}=0}^{\ell} \int_{t_{1} \in \mathbb{R}}\left(\int_{\tau=1}^{\infty} e^{i \tau\left(t-t_{1}\right)} e^{-\frac{1}{2} i\left(x_{2}-x_{1}\right) \tau^{1 / 3}} e^{-\frac{\sqrt{3}}{2}\left(x_{1}+x_{2}\right) \tau^{1 / 3}} \tau^{2 r} d \tau\right) Q\left(x_{2}, t_{1}\right) d t_{1} d x_{2} .
\end{aligned}
$$

Therefore, applying Fubini's theorem and using Hölder's inequality, from (5.36), we get

$$
\begin{aligned}
& \left\|\int_{\tau=1}^{\infty} \int_{x_{2}=0}^{\ell} e^{i \tau t} e^{i a \tau^{1 / 3} x_{2}-i \bar{a} \tau^{1 / 3} x_{1}} \tau^{2 r} \widehat{Q}^{t}\left(x_{2}, \tau\right) d x_{2} d \tau\right\|_{L_{t}^{q}\left[\mathbb{R}: L_{x}^{p}(0, \ell)\right]} \\
\leqslant & \left\|\int_{t_{1} \in \mathbb{R}}\right\| \int_{\tau=1}^{\infty} e^{i \tau\left(t-t_{1}\right)} e^{-\frac{1}{2} i\left(x_{2}-x_{1}\right) \tau^{1 / 3}} e^{-\frac{\sqrt{3}}{2}\left(x_{1}+x_{2}\right) \tau^{1 / 3}} \tau^{2 r} d \tau\left\|_{L_{x_{2}}^{p}(0, \ell) L_{x_{1}}^{p}(0, \ell)}\right\| Q\left(t_{1}\right)\left\|_{L_{x_{2}}^{p^{\prime}}(0, \ell)} d t_{1}\right\|_{L_{t}^{q}(\mathbb{R})} .
\end{aligned}
$$

At this point, we shall need the following lemma for the interval $(0, \ell)$, whose proof is similar to Lemma 2.1 in Ref. 37 for the line. Also, a simple modification of its proof works for the half-line.

Lemma 5.3. The following estimate holds:

$$
\left\|\int_{\tau=1}^{\infty} e^{i \tau t} e^{-\frac{1}{2} i\left(x_{2}-x_{1}\right) \tau^{1 / 3}} e^{-\frac{\sqrt{3}}{2}\left(x_{1}+x_{2}\right) \tau^{1 / 3}} \tau^{-\frac{1}{2}} d \tau\right\|_{L_{x_{2}}^{\infty}(0, \ell) L_{x_{1}}^{\infty}(0, \ell)} \lesssim|t|^{-\frac{1}{2}} .
$$

Applying the above lemma to inequality (5.37), we get

$$
\begin{aligned}
& \left\|\int_{\tau=1}^{\infty} \int_{x_{2}=0}^{\ell} e^{i \tau t} e^{i a \tau^{1 / 3} x_{2}-i \bar{a} \tau^{1 / 3} x_{1}} \tau^{2 r} \widehat{Q}^{t}\left(x_{2}, \tau\right) d x_{2} d \tau\right\|_{L_{t}^{q}\left[\mathbb{R}: i_{x}^{p}(0, \ell)\right]} \\
\leqslant & \left(\int_{t=-\infty}^{\infty}\left|\int_{t_{1}=-\infty}^{\infty}\right| t-\left.\left.t_{1}\right|^{-\frac{1}{2}}\left\|Q\left(\cdot, t_{1}\right)\right\|_{p^{\prime}} d t_{1}\right|^{q} d t\right)^{1 / q} .
\end{aligned}
$$

Finally, using fractional integration, we obtain the desired inequality (5.33).

\section{WELL-POSEDNESS OF KdV IBVP IN SOBOLEV SPACES ON AN INTERVAL}

Finally, having established the key estimate (5.12) in the solution space $X$, now we are ready to prove Theorem 1.1 for the KdV IBVP (1.1).

Existence and uniqueness of solution. We show that the iteration map for the solution of the $\operatorname{KdV} \operatorname{IBVP}(1.1) u \mapsto \Phi u \doteq S\left[u_{0}, g_{0}, h_{0}, h_{1} ; u u_{x}\right]$ is a contraction in the space $X$. First, we show that it is onto. For $u \in X$ and $s \in\left(\frac{3}{4}, 1\right)$, using the estimate $(5.12)$, and for $0<T^{*} \leqslant T<1$, we have

$$
\|\Phi u\|_{X} \leqslant c_{s}\left(\left\|u_{0}\right\|_{H_{x}^{s}(0, \ell)}+\left\|g_{0}\right\|_{H_{t}^{\frac{s+1}{3}}(0, T)}+\left\|h_{0}\right\|_{H_{t}^{\frac{s+1}{3}}(0, T)}+\left\|h_{1}\right\|_{H_{t}^{\frac{s}{3}}(0, T)}+2(1+\sqrt{\ell}) T^{* \frac{1}{3}}\|u\|_{X}^{2}\right) .
$$

Now, choosing $u$ in the ball $B(0, r)=\left\{u \in X:\|u\|_{X} \leqslant r\right\}$ with radius

$$
r \doteq 2 c_{s}\left\|\left(u_{0}, g_{0}, h_{0}, h_{1}\right)\right\|_{D},
$$


where the data norm $\|\cdot\|_{D}$ is defined by Eq. (1.14), and using estimate (6.1), we see that for the iteration map $\Phi$ to be onto it suffices to choose a $T^{*}$ such that $\frac{r}{2}+c_{s}(1+\sqrt{\ell}) T^{* \frac{1}{3}} r^{2} \leqslant r$ or

$$
T^{*} \leqslant\left[64 c_{s}^{6}(1+\sqrt{\ell})^{3}\left\|\left(u_{0}, g_{0}, h_{0}, h_{1}\right)\right\|_{D}^{2}\right]^{-1}
$$

Next, we show that the map $\Phi$ is a contraction in $X$; i.e., for any $u_{1}, u_{2} \in X$, we have

$$
\left\|\Phi u_{1}-\Phi u_{2}\right\|_{X} \leqslant \frac{1}{2}\left\|u_{1}-u_{2}\right\|_{X}
$$

For this, using the identity $u_{1} \partial_{x} u_{1}-u_{2} \partial_{x} u_{2}=\frac{1}{2}\left[\partial_{x}\left(u_{1}+u_{2}\right)\left(u_{1}-u_{2}\right)+\left(u_{1}+u_{2}\right) \partial_{x}\left(u_{1}-u_{2}\right)\right]$ and applying estimate (5.12), we get, for $\frac{3}{4}<s<1$,

$$
\left\|\Phi u_{1}-\Phi u_{2}\right\|_{X}=S\left[0,0,0,0 ;-u_{1} \partial_{x} u_{1}+u_{2} \partial_{x} u_{2}\right] \leqslant 2 c_{s}(1+\sqrt{\ell}) T^{* \frac{1}{3}}\left(\left\|u_{1}\right\|_{X}+\left\|u_{2}\right\|_{X}\right)\left\|u_{1}-u_{2}\right\|_{X}
$$

which gives contraction if $2 c_{s}(1+\sqrt{\ell}) T^{* \frac{1}{3}}(r+r)<1 / 2$, or

$$
T^{*} \leqslant\left[16^{3} c_{s}^{6}(1+\sqrt{\ell})^{3}\left\|\left(u_{0}, g_{0}, h_{0}, h_{1}\right)\right\|_{D}^{3}\right]^{-1} .
$$

Combining conditions (6.3) and (6.5), we get the maximal lifespan

$$
T^{*}=\min \left\{T,\left[16^{3} c_{s}^{6}(1+\sqrt{\ell})^{3}\left\|\left(u_{0}, g_{0}, h_{0}, h_{1}\right)\right\|_{D}^{3}\right]^{-1}\right\}
$$

for which the iteration map $\Phi$ is a contraction in the ball $B(0, r) \subset X$. Thus, the equation $u=\Phi u$ has a unique solution $u \in B(0, r) \subset X$.

Continuity of the data-to-solution map. Let $\left(u_{0}, g_{0}, h_{0}, h_{1}\right)$ and $\left(\tilde{u}_{0}, \tilde{g}_{0}, \tilde{h}_{0}, \tilde{h}_{1}\right)$ be two different data lying inside a ball $B_{\rho} \subset D$ of radius $\rho>$ 0 centered at a distance $r$ from the origin. Furthermore, denote the corresponding solutions to the $\operatorname{KdV} \operatorname{IBVP}(1.1)$ by $u=\Phi_{u_{0}, g_{0}, h_{0}, h_{1}} u$ and $\tilde{u}=\Phi_{\tilde{u}_{0}, \tilde{g}_{0}, \tilde{h}_{0}, \tilde{h}_{1}} \tilde{u}$, respectively. Then, by the contraction condition (6.6), their maximal lifespans $T_{u}$ and $T_{\tilde{u}}$ are given by

$$
T_{u}=\min \left\{T,\left[16^{3} c_{s}^{6}(1+\sqrt{\ell})^{3}\left\|\left(u_{0}, g_{0}, h_{0}, h_{1}\right)\right\|_{D}^{3}\right]^{-1}\right\}, T_{\tilde{u}}=\min \left\{T,\left[16^{3} c_{s}^{6}(1+\sqrt{\ell})^{3}\left\|\left(\tilde{u}_{0}, \tilde{g}_{0}, \tilde{h}_{0}, \tilde{h}_{1}\right)\right\|_{D}^{3}\right]^{-1}\right\} .
$$

Furthermore, observing that the following lifespan

$$
T_{c} \doteq \min \left\{T,\left[16^{3} c_{s}^{6}(1+\sqrt{\ell})^{3}(r+\rho)^{3}\right]^{-1}\right\} \leqslant \min \left\{T_{u}, T_{\tilde{u}}\right\}
$$

is common, we have that both solutions $u$ and $\tilde{u}$ exist for any $0<t \leqslant T_{c}$. Also, if we denote by $X_{T_{u}}$ the solution space $X$ defined by (1.15) with $T^{*}=T_{u}, X_{\tilde{u}}$ the solution space $X$ defined by (1.15) with $T^{*}=T_{\tilde{u}}$, and $X_{c}$ the solution space $X$ defined by $(1.15)$ with $T^{*}=T_{c}$, then we have that $X_{u} \subset X_{c}$ and $X_{\tilde{u}} \subset X_{c}$. Next, we determine a ball $B\left(0, r_{c}\right) \subset X$ such that for any $u, \tilde{u} \in B\left(0, r_{c}\right)$ with data in the ball $B_{\rho}$ we have

$$
\|u-\tilde{u}\|_{X_{c}} \leqslant 2 c_{s}\left\|\left(u_{0}, g_{0}, h_{0}, h_{1}\right)-\left(\tilde{u}_{0}, \tilde{g}_{0}, \tilde{h}_{0}, \tilde{h}_{1}\right)\right\|_{D}
$$

For this, using the estimate (5.12), we have

$$
\begin{aligned}
\|u-\tilde{u}\|_{X_{c}} & =\left\|S\left[u_{0}-\tilde{u}_{0}, g_{0}-\tilde{g}_{0}, h_{0}-\tilde{h}_{0}, h_{1}-\tilde{h}_{1} ; u u_{x}-\tilde{u} \tilde{u}_{x}\right]\right\|_{X_{c}} \\
& \leqslant c_{s}\left\|\left(u_{0}, g_{0}, h_{0}, h_{1}\right)-\left(\tilde{u}_{0}, \tilde{g}_{0}, \tilde{h}_{0}, \tilde{h}_{1}\right)\right\|_{D}+2 c_{s}(1+\sqrt{\ell}) T_{c}^{\frac{1}{3}}\left(\|u\|_{X_{c}}+\|\tilde{u}\|_{X_{c}}\right)\|u-\tilde{u}\|_{X_{c}}
\end{aligned}
$$

Furthermore, since $u, \tilde{u} \in B\left(0, r_{c}\right) \subset X_{c}$, the above inequality implies

$$
\|u-\tilde{u}\|_{X_{c}} \leqslant c_{s}\left\|\left(u_{0}, g_{0}, h_{0}, h_{1}\right)-\left(\tilde{u}_{0}, \tilde{g}_{0}, \tilde{h}_{0}, \tilde{h}_{1}\right)\right\|_{D}+2 c_{s}(1+\sqrt{\ell}) T_{c}^{\frac{1}{3}} \cdot 2 r_{c} \cdot\|u-\tilde{u}\|_{X_{c}},
$$

which after combining the terms $\|u-\tilde{u}\|_{X_{c}}$ gives the following choice of $r_{c}$ :

$$
r_{c}=\frac{1}{4 c_{s} \sqrt{T_{c}}}=\max \left\{\left[8 c_{s}(1+\sqrt{\ell}) T_{c}^{\frac{1}{3}}\right]^{-1}, 2 c_{s}(r+\rho)\right\}
$$

which implies (6.8), i.e., the Lipschitz continuity of the data-to-solution map. 


\section{DERIVATION OF THE FOKAS SOLUTION FORMULA}

In this section, we derive the UTM formula for the linear $\mathrm{KdV}$ on the interval

$$
\begin{aligned}
& \partial_{t} u+\partial_{x}^{3} u=f, \quad 0<x<\ell, 0<t<T, \\
& u(x, 0)=u_{0}(x), \\
& u(0, t)=g_{0}(t), \quad u(\ell, t)=h_{0}(t), \quad u_{x}(\ell, t)=h_{1}(t) .
\end{aligned}
$$

Using the adjoint equation $\partial_{t} \tilde{u}+\partial_{x}^{3} \tilde{u}=0$ for $\tilde{u}=e^{-i k x-i k^{3} t}$, we get the divergence form

$$
\left(e^{-i k x-i k^{3} t} u\right)_{t}+\left(e^{-i k x-i k^{3} t}\left[\partial_{x}^{2} u+i k \partial_{x} u-k^{2} u\right]\right)_{x}=e^{-i k x-i k^{3} t} f .
$$

Defining the Fourier transform for an $L^{2}$-function $\varphi(x)$ on the interval $(0, \ell)$ by

$$
\hat{\varphi}(k) \doteq \int_{0}^{\ell} e^{-i k x} \varphi(x) d x, \quad k \in \mathbb{C},
$$

we integrate the divergence form (7.2) over $[0, \ell]$ and solve the first order differential equation with respect to $t$ to obtain the following global relation:

$$
\begin{aligned}
e^{-i k^{3} t} \hat{u}(k, t)= & \hat{u}_{0}(k)+F(k, t)+\left[\tilde{g}_{2}\left(k^{3}, t\right)+i k \tilde{g}_{1}\left(k^{3}, t\right)-k^{2} \tilde{g}_{0}\left(k^{3}, t\right)\right] \\
& -e^{-i k \ell}\left[\tilde{h}_{2}\left(k^{3}, t\right)+i k \tilde{h}_{1}\left(k^{3}, t\right)-k^{2} \tilde{h}_{0}\left(k^{3}, t\right)\right], \quad k \in \mathbb{C},
\end{aligned}
$$

where

$$
F(k, t) \doteq \int_{0}^{t} e^{-i k^{3} \tau} \hat{f}(k, \tau) d \tau, \quad \tilde{g}_{j}(k, t) \doteq \int_{0}^{t} e^{-i k \tau} \partial_{x}^{j} u(0, \tau) d \tau, \quad \tilde{h}_{j}(k, t) \doteq \int_{0}^{t} e^{-i k \tau} \partial_{x}^{j} u(\ell, \tau) d \tau .
$$

Inverting equation (7.4), we find the integral representation

$$
\begin{aligned}
u(x, t)= & \frac{1}{2 \pi} \int_{-\infty}^{\infty} e^{i k x+i k^{3} t}\left[\hat{u}_{0}(k)+F(k, t)\right] d k+\frac{1}{2 \pi} \int_{-\infty}^{\infty} e^{i k x+i k^{3} t}\left[\tilde{g}_{2}\left(k^{3}, t\right)+i k \tilde{g}_{1}\left(k^{3}, t\right)-k^{2} \tilde{g}_{0}\left(k^{3}, t\right)\right] d k \\
& -\frac{1}{2 \pi} \int_{-\infty}^{\infty} e^{i k(x-\ell)+i k^{3} t}\left[\tilde{h}_{2}\left(k^{3}, t\right)+i k \tilde{h}_{1}\left(k^{3}, t\right)-k^{2} \tilde{h}_{0}\left(k^{3}, t\right)\right] d k,
\end{aligned}
$$

which involves the unknown boundary values $\partial_{x} u(0, t), \partial_{x}^{2} u(0, t), \partial_{x}^{2} u(\ell, t)$ via the transforms $\tilde{g}_{1}, \tilde{g}_{2}, \tilde{h}_{2}$. To eliminate these unknowns, we deform the contours of integration from $\mathbb{R}$ to the boundaries of the regions $D^{+}, D_{1}^{-}$, and $D_{2}^{-}$(see Fig. 1 ) and hence rewrite (7.5) as follows:

$$
\begin{aligned}
u(x, t)= & \frac{1}{2 \pi} \int_{-\infty}^{\infty} e^{i k x+i k^{3} t}\left[\hat{u}_{0}(k)+F(k, t)\right] d k+\frac{1}{2 \pi} \int_{\partial D^{+}} e^{i k x+i k^{3} t}\left[\tilde{g}_{2}\left(k^{3}, t\right)+i k \tilde{g}_{1}\left(k^{3}, t\right)-k^{2} \tilde{g}_{0}\left(k^{3}, t\right)\right] d k \\
& +\frac{1}{2 \pi} \int_{\partial D_{1}^{-}} e^{i k(x-\ell)+i k^{3} t}\left[\tilde{h}_{2}\left(k^{3}, t\right)+i k \tilde{h}_{1}\left(k^{3}, t\right)-k^{2} \tilde{h}_{0}\left(k^{3}, t\right)\right] d k \\
& +\frac{1}{2 \pi} \int_{\partial D_{2}^{-}} e^{i k(x-\ell)+i k^{3} t}\left[\tilde{h}_{2}\left(k^{3}, t\right)+i k \tilde{h}_{1}\left(k^{3}, t\right)-k^{2} \tilde{h}_{0}\left(k^{3}, t\right)\right] d k .
\end{aligned}
$$

The above deformation is possible, thanks to analyticity in $k$ (Cauchy's theorem) and the exponential decay of the relevant integrands in the complements of the regions $D^{+}, D_{1}^{-}$, and $D_{2}^{-}$. For example,

$$
\begin{aligned}
& \int_{-\infty}^{\infty} e^{i k x+i k^{3} t}\left[\tilde{g}_{2}\left(k^{3}, t\right)+i k \tilde{g}_{1}\left(k^{3}, t\right)-k^{2} \tilde{g}_{0}\left(k^{3}, t\right)\right] d k \\
= & \int_{\partial D^{+}} e^{i k x+i k^{3} t}\left[\tilde{g}_{2}\left(k^{3}, t\right)+i k \tilde{g}_{1}\left(k^{3}, t\right)-k^{2} \tilde{g}_{0}\left(k^{3}, t\right)\right] d k
\end{aligned}
$$

since (i) $e^{i k x}$ is bounded for $\operatorname{Im}(k) \geqslant 0$ (recall that $x \geqslant 0$ ) and exponentially decaying for $\operatorname{Im}(k)>0$ and (ii) $e^{i k^{3}(t-\tau)}$ is bounded for $\operatorname{Im}\left(k^{3}\right) \geqslant 0$ (recall that $t \geqslant \tau$ ), i.e., for $\arg (k) \in\left(0, \frac{\pi}{3}\right) \cup\left(\frac{\pi}{3}, \pi\right) \cup\left(\frac{4 \pi}{3}, 2 \pi\right)$, and exponentially decaying for $\operatorname{Im}\left(k^{3}\right)>0$, and hence, $(-\infty, \infty)$ can be deformed to the boundary of the region $D^{+}$via Cauchy's theorem. The rest of the terms in (7.5) and (7.6) can be handled like (7.7). 
Next, combining the global relation (7.4) with the two identities obtained from it after replacing $k$ by $\alpha k$ and $\alpha^{2} k$ with $\alpha=e^{\frac{2 \pi}{3}}$, we get a system of three equations that are solved via Cramer's rule for the unknown functions $\tilde{g}_{1}, \tilde{g}_{2}, \tilde{h}_{2}$. Substituting the resulting expressions into formula (7.6), we get the desired UTM formula (1.18). Crucial to this last step of the computation is the following fact.

Lemma 7.1. The zeros of $\Delta(k)$ lie outside $D^{+} \cup D_{1}^{-} \cup D_{2}^{-}$. More precisely, all the zeros lie along the rays $e^{\frac{\pi}{6} i}, e^{\frac{3 \pi}{2} i}, e^{\frac{5 \pi}{6} i}$.

\section{ACKNOWLEDGMENTS}

The first author was partially supported by a grant from the Simons Foundation (No. 524469 to Alex Himonas). The authors would like to thank the referee of the paper for constructive comments.

\section{REFERENCES}

' J. L. Bona and J.-C. Saut, “Dispersive blow-up of solutions of generalised Korteweg-de Vries equations,” J. Differ. Equations 103, 3-57 (1993).

${ }^{2}$ J. L. Bona and R. W. Smith, "The initial-value problem for the Korteweg-de Vries equation," Philos. Trans. R. Soc., A 278, 555-601 (1975).

${ }^{3}$ J. L. Bona, S. M. Sun, and B.-Y. Zhang, “A non-homogeneous boundary-value problem for the Korteweg-de Vries equation in a quarter plane," Trans. Amer. Math. Soc. 354(2), 427-490 (2002).

${ }^{4}$ J. L. Bona, S. M. Sun, and B.-Y. Zhang, "A nonhomogeneous boundary-value problem for the Korteweg-de Vries equation posed on a finite domain," Commun. Partial. Differ. Equations 28(7-8), 1391-1436 (2003).

${ }^{5}$ J. L. Bona, S. M. Sun, and B.-Y. Zhang, "Boundary smoothing properties of the Korteweg-de Vries equation in a quarter plane and applications," Dyn. Partial Differ. Equations 3(1), 1-69 (2006).

${ }^{6}$ J. L. Bona, S. M. Sun, and B.-Y. Zhang, "Nonhomogeneous boundary value problems of one-dimensional nonlinear Schrödinger equations," J. Math. Pures Appl. 109, 1-66 (2018).

${ }^{7}$ J. L. Bona and R. Winther, “The Korteweg-de Vries equation, posed in a quarter-plane," SIAM J. Math. Anal. 14(6), 1056-1106 (1983).

${ }^{8}$ J. Bourgain, "Fourier transform restriction phenomena for certain lattice subsets and applications to nonlinear evolution equations. Part II: The KdV equation," Geom. Funct. Anal. 3(3), 209-262 (1993).

${ }^{9}$ J. Bourgain, "Fourier transform restriction phenomena for certain lattice subsets and applications to nonlinear evolution equations," Geom. Funct. Anal. 3(2), 107-157 (1993).

${ }^{10}$ J. V. Boussinesq, Essai Sur la Théorie des Eaux Courantes, Mémoires présentés par divers savants à l'Académie des Sciences (Imprimerie Nationale, 1877), Vol. 23, pp. $1-680$.

${ }^{11}$ M. Christ, J. Colliander, and T. Tao, “Asymptotics, frequency modulation, and low regularity ill-posedness for canonical defocusing equations," Am. J. Math. 125(6), 1235-1293 (2003).

${ }^{12}$ J. Colliander, M. Keel, G. Staffilani, H. Takaoka, and T. Tao, “Sharp global well posedness for KdV and modified KdV on $\mathbb{R}$ and T," J. Amer. Math. Soc. 16(3), 705-749 (2003).

${ }^{13}$ J. E. Colliander and C. E. Kenig, "The generalised Korteweg-de Vries equation on the half-line,” Commun. Partial. Differ. Equations 27(11-12), 2187-2266 (2002).

${ }^{14}$ P. Constantin and J.-C. Saut, "Local smoothing properties of dispersive equations," J. AMS 1(2), 413-439 (1988).

${ }^{15}$ B. Deconinck, T. Trogdon, and V. Vasan, "The method of Fokas for solving linear partial differential equations," SIAM Rev. 56(1), 159-186 (2014).

${ }^{16}$ A. V. Faminskii, "A mixed problem in a semistrip for the Korteweg-de Vries equation and its generalisations," Dinamika Sploshn. Sredy 258, 54-94 (1988) [Moscow Math. Soc. 1989, 53-91].

${ }^{17}$ A. S. Fokas, “A unified transform method for solving linear and certain nonlinear PDEs," Proc. R. Soc. London, Ser. A 453, 1411-1443 (1997).

${ }^{18}$ A. S. Fokas, "A new transform method for evolution partial differential equations," IMA J. Appl. Math. 67, 559-590 (2002).

${ }^{19}$ A. S. Fokas, A Unified Approach to Boundary Value Problems (SIAM, 2008).

${ }^{20}$ A. S. Fokas, A. Himonas, and D. Mantzavinos, “The Korteweg-de Vries equation on the half-line," Nonlinearity 29, 489-527 (2016).

${ }^{21}$ A. S. Fokas, A. Himonas, and D. Mantzavinos, "The nonlinear Schrödinger equation on the half-line," Trans. Am. Math. Soc. 369, 681-709 (2017).

${ }^{22}$ A. S. Fokas and A. Its, "The nonlinear Schrödinger equation on the interval," J. Phys. A: Math. Gen. 37, 6091-6114 (2004).

${ }^{23}$ A. S. Fokas, A. Its, and L.-Y. Sung, "The nonlinear Schrödinger equation on the half-line," Nonlinearity 18, 1771-1822 (2005).

${ }^{24}$ A. S. Fokas and B. Pelloni, "A transform method for linear evolution PDEs on a finite interval," IMA J. Appl. Math. 70, 564-587 (2005).

${ }^{25}$ A. S. Fokas and B. Pelloni, Unified Transform for Boundary Value Problems: Applications and Advances (SIAM, 2015).

${ }^{26}$ A. S. Fokas and E. Spence, "Synthesis, as opposed to separation, of variables," SIAM Rev. 54, 291-324 (2012).

${ }^{27}$ C. S. Gardner, J. M. Greene, M. D. Kruskal, and R. M. Miura, "Method for solving the Korteweg-de Vries equation," Phys. Rev. Lett. 19(19), 1095-1097 (1967).

${ }^{28}$ Z. Guo, "Global well-posedness of Korteweg-de Vries equation in $H^{-3 / 4}(\mathbb{R})$," J. Math. Pures Appl. 91(6), 583-597 (2009).

${ }^{29}$ G. H. Hardy, "The constants of certain inequalities," Journal London Math. Soc. 8(2), 114-119 (1933).

${ }^{30}$ A. Himonas and D. Mantzavinos, “The 'good' Boussinesq equation on the half-line," J. Differ. Equations 258, 3107-3160 (2015).

${ }^{31}$ J. Holmer, "The initial-boundary-value problem for the 1D nonlinear Schrödinger equation on the half-line," Differ. Integr. Equations 18, 647-668 (2005).

${ }^{32}$ J. Holmer, "The initial-boundary-value problem for the Korteweg-de Vries equation," Commun. Partial. Differ. Equations 31, 1151-1190 (2006).

${ }^{33}$ C. E. Kenig, G. Ponce, and L. Vega, "Well-posedness of the initial value problem for the Korteweg-de Vries equation," J. AMS 4, 323-347 (1991).

${ }^{34}$ C. E. Kenig, G. Ponce, and L. Vega, “A bilinear estimate with applications to the KdV equation,” J. Amer. Math. Soc. 9(2), 571-603 (1996).

${ }^{35}$ C. E. Kenig, G. Ponce, and L. Vega, "Well-posedness and scattering results for the generalised Korteweg-de Vries equation via the contraction principle," Commun. Pure Appl. Math. 46(4), 527-620 (1993).

${ }^{36}$ C. E. Kenig, G. Ponce, and L. Vega, “On the ill-posedness of some canonical dispersive equations," Duke Math. J. 106(3), 617-633 (2001).

${ }^{37}$ C. E. Kenig, G. Ponce, and L. Vega, “On the (generalised) Korteweg-de Vries equation,” Duke Math. J. 59(3), 585-610 (1989). 
${ }^{38}$ D. J. Korteweg and G. de Vries, "On the change of form of long waves advancing in a rectangular canal, and on a new type of long stationary waves," Philos. Mag. 39(240), 422-443 (1895).

${ }^{39}$ P. D. Lax, "Integrals of nonlinear equations of evolution and solitary waves," Commun. Pure Appl. Math. 21, 467-490 (1968).

${ }^{40}$ J. Lenells, "The KdV equation on the half-line: The Dirichlet to Neumann map," J. Phys. A: Math. Theor. 46, 345203 (2013).

${ }^{41}$ F. Linares and G. Ponce, Introduction to Nonlinear Dispersive Equations (Universitext Springer, New York, 2009).

${ }^{42}$ W. McLean, Strongly Elliptic Systems and Boundary Integral Equations (Cambridge University Press, 2000).

${ }^{43}$ T. Özsari and N. Yolcu, "The initial-boundary value problem for the biharmonic Schrödinger equation on the half-line," Commun. Pure Appl. Anal. (to be published); preprint arXiv:1802.10499.

${ }^{44}$ J.-C. Saut and R. Temam, "Remarks on the Korteweg-de Vries equation," Isr. J. Math. 24(1), 78-87 (1976).

${ }^{45} \mathrm{~J}$. Scott Russell, "Report on waves," in Report of the 14th Meeting of the British Association for the Advancement of Science, London, 1845.

${ }^{46}$ A. Sjöberg, "On the Korteweg-de Vries equation: Existence and uniqueness," J. Math. Anal. Appl. 29, 569-579 (1970).

${ }^{47}$ B. A. Ton, "Initial boundary-value problems for the Korteweg-de Vries equation," J. Differ. Equations 25, 288-309 (1977).

${ }^{48}$ N. J. Zabusky and M. D. Kruskal, "Interaction of solitons in a collisionless plasma and the recurrence of initial states," Phys. Rev. Lett. 15(6), 240-243 (1965). 